\title{
A stochastic computational multiscale approach; Application to MEMS resonators
}

\author{
V. Lucas ${ }^{\mathrm{a}}$, J.-C. Golinval ${ }^{\mathrm{a}}$, S. Paquay ${ }^{\mathrm{b}}$, V.-D. Nguyen ${ }^{\mathrm{a}}$, L. Noels ${ }^{\mathrm{a}, *}$, L. $\mathrm{Wu}^{\mathrm{a}}$ \\ ${ }^{a}$ University of Liege, Department of Aeronautics and Mechanical Engineering \\ Chemin des Chevreuils 1, B-4000 Liège, Belgium \\ ${ }^{b}$ Open-Engineering SA, Rue des Chasseurs-Ardennais, B-4031, Liège (Angleur), Belgium
}

\begin{abstract}
The aim of this work is to develop a stochastic multiscale model for polycrystalline materials, which accounts for the uncertainties in the micro-structure. At the finest scale, we model the micro-structure using a random Voronoï tessellation, each grain being assigned a random orientation. Then, we apply a computational homogenization procedure on statistical volume elements to obtain a stochastic characterization of the elasticity tensor at the meso-scale. A random field of the meso-scale elasticity tensor can then be generated based on the information obtained from the SVE simulations. Finally, using a stochastic finite element method, these meso-scale uncertainties are propagated to the coarser scale. As an illustration we study the resonance frequencies of MEMS micro-beams made of poly-silicon materials, and we show that the stochastic multiscale approach predicts results in agreement with a Monte Carlo analysis applied directly on the fine finite-element model, i.e. with an explicit discretization of the grains.
\end{abstract}

Keywords: Multi-scale, Stochastic, Finite elements, Polycrystalline, Resonance frequency, MEMS

\section{Introduction}

Uncertainty is an inherent nature of materials, especially when they are heterogeneous. The heterogeneity of a material has a significant impact on its properties and might influence the response of structures made of that material as well. The uncertainty due to the heterogeneous nature of the material can be described as the spatial variability of the material properties in the structure. When considering a finite element analysis, the structural response variability can be predicted using the direct Monte Carlo method, which can lead to an overwhelming computation cost as it involves the finite-element discretization of

\footnotetext{
*Corresponding author, Phone: +32 436648 26, Fax: +32 43669505

Email address: L.Noels@ulg.ac.be (L. Noels)
}

Preprint submitted to Computer Methods in Applied Mechanics and EngineeringApril 18, 2015 
the heterogeneities. In order to solve the problem of structural stochasticity at a reasonable computation cost, Stochastic Finite Element (SFE) analyzes were developed [1-3].

In the context of stochastic finite element analyzes, a random field, which is used to describe the heterogeneity of a material, is discretized in accordance with the finite element mesh. The proper mesh size depends primarily on the standard deviation and correlation length of the random field, which constrains the variation of the random variable within each element to be small enough [4]. Therefore finite element sizes smaller than the correlation length, such as one half of the correlation length [5], are required to ensure the accuracy of the analysis results. In all generalities, the correlation length of the random material property depends on the characteristic length of the material heterogeneity. As the interest of this work focuses on polycrystalline materials, the correlation length is related to the size of the grains, meaning that each grain needs to be meshed in a conformed way. With such a method, the analysis of the structural stochasticity is very expensive in terms of computational resources. Moreover using a finite element discretization based on the explicit micro-grain structure leads to a noise field [6] instead of a smooth one [7]. Therefore the stochastic finite element method, such as the Neumann expansion [8] or perturbation approximation [9], cannot be applied. To achieve a reduction of the computational cost of a structural stochasticity analysis, we seek the recourse to multi-scale computational methods in which a smooth random field is defined at the meso-scale.

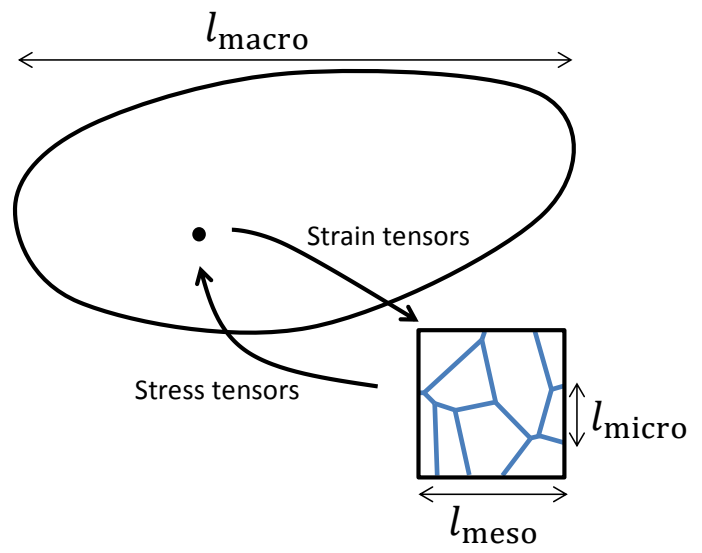

Figure 1: The different scales involved in a multiscale analysis

Multi-scale methods were developed with the rise of structural applications made of heterogeneous materials such as composite materials, metal alloys, polycrystalline materials... In a multi-scale method, the macro-scale or structuralscale behavior can be related to the micro-scale properties, through a homogenization technique. The different scales involved in such an analysis, which are 
depicted in Fig. 1, are

- The micro-scale: is the characteristic size of the micro-structure, such as the size of inclusions for composite materials or the size of grains for polycrystalline materials, and is denoted by $l_{\text {micro }}$;

- The meso-scale: is an intermediate scale, such as the size of the volume element over which the homogenization is performed, and is denoted by $l_{\text {meso }}$;

- The macro-scale: also called structural scale, is the size of the structural problem, and is denoted by $l_{\text {macro }}$.

Homogenization methods relate the macroscopic strain tensor to the macroscopic stress tensor through the resolution of a meso-scale boundary value problem (BVP). In this framework, the structural-scale BVP is seen as a continuum homogeneous medium and the meso-scale BVP contains the different sources of heterogeneities. To this end, the meso-scale BVP is defined on a Representative Volume Element (RVE), which represents the micro-structure and the micro-structural behavior in a statistically representative way.

The homogenization process on the RVE can be conducted in a semi-analytical way, as for the mean-field-homogenization method for which the average phase-fields of the meso-scale problem are considered in order to derive the macro-response, see for example [10-12] for a state-of-the-art review. The mesoscale BVP can also be solved, for more general constitutive behaviors and microstructures, in a numerical way by using a voxelization of the micro-structure as in the Fast-Fourier-Transform method introduced in [13] or a finite element discretization of the micro-structure as in the computational homogenization, also called $\mathrm{FE}^{2}$, method. Early developments of this last approach were proposed in [14] with the VCFEM (Voronoï Cell Finite Element Method). More recent developments can be found in the works of [15-17], as an non-exhaustive list. In the context of computational homogenization methods, the meso-scale finite element problem is solved by applying on the RVE adequate Boundary Conditions (BCs) which should satisfy the Hill-Mandel condition stating that the deformation energy at the macroscopic level should be equal to the volume average of the micro-scale stress work. To be called strictly representative a RVE should be defined so that the homogenized results are not dependent on the (energetically consistent) BCs, although the use of periodic boundary conditions (PBCs) allows using meso-scale volume elements of smaller sizes as the convergence rate of the homogenized properties with respect to the RVE size is faster than with other BCs $[17,18]$. A review of multi-scale computational homogenization can be found in reference [19]. Finally, the BVPs at the different scales, possibly more than two, can also be defined using the asymptotic homogenization method, see [20] for an extensive review.

Multi-scale methods have been shown to be efficient and accurate computational tools to model structures made of complex heterogeneous materials. 
Nevertheless, one should keep in mind that they are based on the scales separation assumptions, which read

$$
\begin{aligned}
& l_{\text {meso }}<<l_{\text {macro }}, \text { and } \\
& l_{\text {micro }}<<l_{\text {meso }} .
\end{aligned}
$$

On the one hand, the first equation (1) states that, in order to be accurate, the size of the meso-scale volume element on which the homogenization is applied should be smaller than the characteristic length on which the macro-scale loading varies in space [19]. This condition should always be satisfied. On the other hand, the second equation (2) states that to be statistically representative, and thus to qualify to be a RVE, the meso-scale volume element should be much larger than the micro-structural size [21].

When the structural-size is not several orders of magnitude larger than the micro-structural size, this second condition (2) cannot be satisfied while respecting the first condition (1). In this case, the volume element at the meso-scale is called Statistical Volume Element (SVE) [22]. Since an SVE is not statistically representative by definition, different homogenized meso-scale responses are obtained when applying different kinds of energetically consistent BCs on the SVE. Moreover, even when considering a unique case of BCs, different homogenized properties are obtained for different SVE realizations of the same size. This means that the uncertainties at the micro-scale should be up-scaled to the structural-scale through the SVE resolutions at the meso-scale. When performing the homogenization though the use of SVEs, the meso-scale length $l_{\text {meso }}$ is also the size of the SVE, $l_{\mathrm{SVE}}$.

In the literature, stochastic multi-scale analyzes have been developed to account for fine-scale material properties as random variables -and random fields in particular cases- by using order reduction of asymptotic homogenization [23]. However in order to account for general fine-scale random fields, the resolution of the micro-scale problems during the resolution of the structural-scale analysis can lead to a prohibitive costs when considering probabilistic studies. This motivates the recourse to the definition of a meso-scale random field to conduct the stochastic finite element method. Computational homogenization methods have been applied on SVEs to define the probability convergence criterion of RVE for masonry [24], and to study the scale-dependency of homogenization for random composite materials [25]. In [26], the simulations of SVEs are used to capture the stochastic properties of the parameters in the constitutive model with which the uncertainties are propagated to the structural scale using the stochastic finite element method [27]. In the case of finite elasticity, the resolution of composite material elementary cells is used to explicitly define a meso-scale potential with the aim of studying the uncertainties in the fibers geometry/distribution [28].

The objective of this work is to develop a 3-scale methodology which can be applied to the stochastic analysis of the mechanical response of small dimension structures made of polycrystalline materials, following the process illustrated in Fig. 2.

At the micro-scale we consider anisotropic materials to represent the grains, 


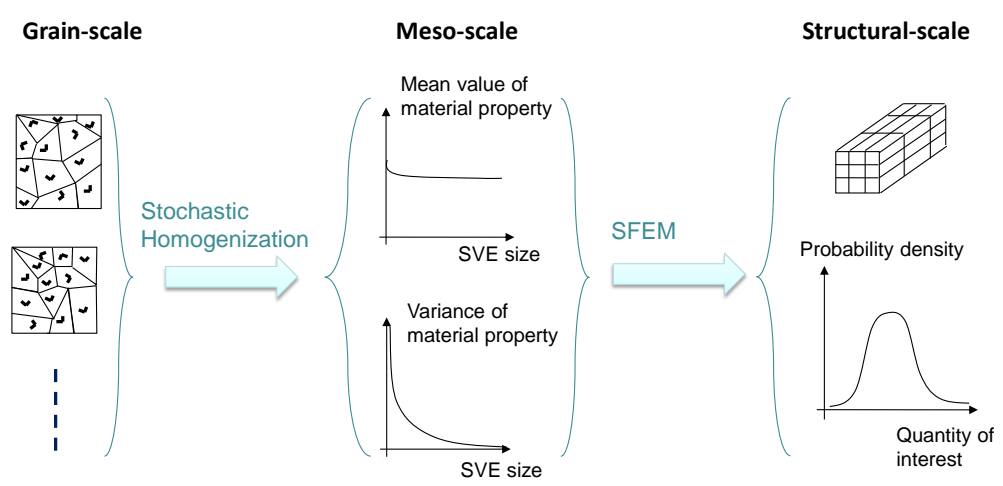

Figure 2: The 3-scale methodology

with different shapes and orientations, of the polycrystalline material.

At the meso-scale finite-element simulations on different SVEs are used to identify the distribution of the homogenized elasticity tensor of the polycrystalline material and the material properties spatial correlations. A random field of the meso-scale elasticity tensor can then be generated based on the information obtained from the SVE simulations. A similar approach can be found in [6], where the so-called moving window technique is used to study the stress concentration due the heterogeneous micro-structure in UD-fiber reinforced composite materials. Since the elasticity tensor takes the form of a positive-definite matrix in its Voigt notation, the generated random meso-scale elasticity tensors need to be consistent with this property. Random positive-definite matrices can be generated by the means of the Cholesky decomposition [8]. In order to ensure the existence of the expectation of the norm of the generated tensor inverses $[29,30]$, we introduce a lower bound, as proposed in [31], when generating the meso-scale material tensors. Bounds were also introduced in the random field generator in the other works $[32,33]$. The generation of the spatially correlated random matrices is achieved by generating a multivariate random field. There are mainly two methods which are widely used to simulate homogeneous random fields. One is the Karhunen-Loeve expansion, which was used to obtain a multivariate random field in the recent work [34]. The other one is the spectral representation, which was developed in $[35,36]$ for multivariate and multidimensional fields. From the discrete correlation functions obtained from the homogenization results of the SVE simulations, the discrete spectral density can be easily computed using a Fast Fourier Transform (FFT) according to the Wiener-Khinchin theorem, motivating the use of the spectral representation in this work. In order for the stochastic multi-scale method to recover the distributions obtained with direct finite element MC simulations, enough SVE realizations ought to be computed to define an accurate meso-scale random field. This motivates the use of the spectral representation generator as a lot of information is available. The spectral representation generates a Gaussian 
field used to define the elasticity tensor field (which is non-Gaussian because of the presence of a lower bound). However, non-Gaussian tensor-valued random fields could also be obtained with the spectral representation method followed by proper mapping techniques, see for example [50], or with the help of the polynomial chaos expansion combined to the Karhunen-Loeve expansion [37]. It is also possible to define a meso-scale random field from a reduced number of micro-samples. For example a posterior model is applied on the first generated random field to improve the accuracy of the generator [37].

Finally, the resulting random field description of the meso-scale material properties can be used with the stochastic finite element method to predict the probabilistic behavior at the structural-scale. With this multi-scale method the meso-scale random field is smooth and has a correlation length larger than when considering explicitly the grain discretization. Hence coarser meshes can be used in the framework of the stochastic finite-element methods, eventually reducing the computation cost.

In order to verify the accuracy and computational efficiency of the stochastic multi-scale method, we study a micro-electro-mechanical systems (MEMS) resonator made of poly-silicon. The uncertainties in the grain size and orientation can lead to a scatter in the device performances, and in particular in the first resonance frequencies. The problem of micro-beams frequency analysis is first solved using a full direct numerical simulation, i.e. for which the grains are meshed, combined to a Monte-Carlo method, which allows the probability description to be computed. This methodology is computationally expensive due to the number of degrees of freedom required to study one sample. The proposed non-deterministic multi-scale strategy is then applied allowing the use of coarser finite elements at the structural-scale and is shown to predict similar probabilistic distributions than the full-scale Monte-Carlo simulations, but at lower computational costs. In the context of the 3-scale method, different SVE sizes and different structural-scale finite element meshes are successively considered to demonstrate that by accounting for the spatial correlation length of the meso-scale homogenized properties, correct predictions are made if the distance between integration points of the finite-element mesh remains smaller than the mesoscopic correlation length.

The organization of the paper is as follows. Stochastic finite element methods are mainstream nowadays and will be briefly recalled in Section 2 . The transition between the micro-scale and the meso-scale, through the resolution of the mesoscale BVPs on the SVEs, is described in Section 3. The generation of the spatially correlated random field of the meso-scale elasticity tensors is detailed in Section 4. Section 5 describes how the resulting meso-scale random field is used in combination with the stochastic finite element method to predict the structural-scale probabilistic behavior. As an illustration case we consider the problem of the MEMS micro-beam resonance analysis. Perspectives and conclusions are drawn in Section 6. 


\section{Stochastic Finite Elements}

The stochastic finite element method (SFEM) is a powerful and widely used tool in computational stochastic mechanics. As an extension of the deterministic FEM, SFEM can solve static and dynamic stochastic problems in which the uncertainties result from the geometry, material, or loading properties. By using finite elements whose properties are random, SFEM can propagate the uncertainties through the mechanical system and assess its stochastic response.

The deterministic problem of an un-damped mechanical structure can be discretized using the finite element method. To this end, the spatial domain $\boldsymbol{D}$ is discretized into the finite elements $\boldsymbol{D}_{i}$, such that $\boldsymbol{D}=\bigcup_{i} \boldsymbol{D}_{i}$, where $\boldsymbol{D}_{i}$ includes the finite element boundary, resulting in the following set of equations

$$
M \ddot{u}+K u=f,
$$

where $\boldsymbol{M}$ and $\boldsymbol{K}$ are respectively the assembled mass and stiffness matrices, $\boldsymbol{f}$ is the assembled external force vector, and $\boldsymbol{u}$ is the assembled vector of the displacement degrees of freedom.

In this work, we consider the uncertainties resulting from the heterogeneous micro-structure, which in turn result in uncertainties in the material properties. The material properties are thus represented by a random field of the elasticity tensor. Let $\boldsymbol{C}(\boldsymbol{x}, \boldsymbol{\theta}): \boldsymbol{D} \times \boldsymbol{\Omega} \rightarrow \boldsymbol{M}_{N}^{+}(R)$ be a random field of the elasticity tensor, in its Voigt notation, over the spatial domain $\boldsymbol{D}$, which is a function of the spatial coordinate $\boldsymbol{x} . \boldsymbol{\theta} \in \boldsymbol{\Omega}$ denotes the elements in the sample space involving random quantities and $\boldsymbol{M}_{N}^{+}(R)$ refers to the set of all symmetric positive-definite real matrices of size $N \times N$.

A SFEM analysis can be defined by the following steps [3]: (i) the discretization of the stochastic fields representing the properties with uncertainties, (ii) the formulation of the stochastic matrices, and (iii) the response variability calculation.

For the first step, i.e. the discretization of the elasticity tensor random field, in order to evaluate the different elementary stiffness matrices of the finite element discretization, the point discretization method is considered in this paper. With the point discretization method, the random field $\boldsymbol{C}(\boldsymbol{x}, \boldsymbol{\theta})$ is evaluated at some integration points $\boldsymbol{x}_{i}$, leading to the random variables $\boldsymbol{C}\left(\boldsymbol{x}_{i}, \boldsymbol{\theta}\right)$. The integration points considered in this work are the mid-points of each finite element (mid-point discretization). As shown in [38], the point discretization method tends to over-represent the uncertainties in each element. Note that other discretization methods, such as the local average method, exist [38]. The point discretization method is easy to implement but its accuracy depends on the mesh discretization: the mesh elements must be small enough compared to the correlation length so that the properties can be considered constant over the mesh elements [39]. The correlation length of a stationary random field is defined by [4]

$$
l_{\mathbf{C}}=\frac{\int_{-\infty}^{\infty} R(\tau) \mathrm{d} \tau}{R(0)},
$$


where $R(\tau)$ is the correlation function of the considered random value.

When considering the second step, i.e the formulation of the stochastic matrices, as the uncertainties result from the elasticity tensor, the stiffness matrix of the finite element discretization is subjected to uncertainties and the nondeterministic problem is thus stated by

$$
M \ddot{u}+K(\theta) u=f .
$$

Finally, to solve the third step, i.e. the response variability calculation, Monte Carlo simulations can be used. To achieve a given accuracy, the system of equations (5) must be solved enough times. The computational cost required to solve this system of equations highly depends on the involved mesh size, which needs to be chosen carefully. As discussed before, there exists a strong link between the spatial correlation length (4) of the random field and the mesh size: the mesh size should be sufficiently small compared to the correlation length. As for a structure made of a polycrystalline material the random field of the material properties corresponds to a noise field, the SFEM described previously cannot be applied directly $[6,7]$ and the structural finite element problem should thus be solved based on the realizations of micro-structures such that each grain is meshed with several finite elements. This motivates the introduction of an intermediate scale, the meso-scale, which represents an aggregate of several grains. At this scale, the random field description for the material properties has a correlation length larger than when considering explicitly the grain discretization, allowing the use of coarser elements in the SFEM at the structural-scale.

\section{Stochastic homogenization: from the micro-scale to the meso-scale}

In this section we detail how to extract the meso-scale random field of the elasticity tensors from the finite element resolution of meso-scale volume elements. First generalities on homogenization methods are recalled, with a particular emphasis on the definition of Representative and Statistical Volume Elements (RVEs and SVEs) and on the definition of effective and apparent meso-scale material tensors. Then we discuss how the apparent material tensor can be evaluated from finite element discretizations of the SVEs. We therefore consider different SVE realizations, with different sizes, from which the distributions of the apparent meso-scale material tensor and its spatial correlation can be extracted. Finally, we apply the method to a poly-silicon material.

\subsection{Generalities on Representative and Statistical Volume Elements}

The aim of this section is to define the scale transition from the microscale up to an intermediate scale: the meso-scale, see Fig. 1. As this work focuses on polycrystalline materials, the micro-scale is characterized by the grain distribution. The grain size is assumed to be large enough $(>100 \mathrm{~nm})$ so that grain boundary mechanics can be neglected. A meso-scale volume element $\omega$ is depicted in Fig. 3. 


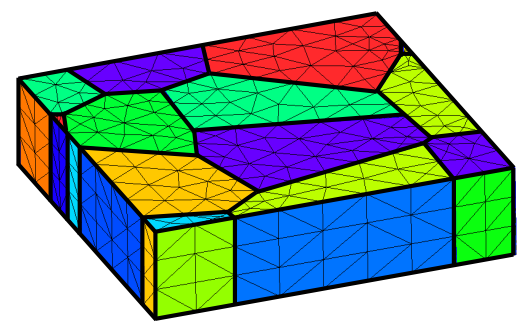

Figure 3: A sample of the meso-scale volume element

Within a multi-scale framework, one can define macro-scale values as the volume average of a micro-scale field on the meso-scale volume-element $\omega$, following

$$
a_{\mathrm{M}}=<a_{\mathrm{m}}>=\frac{1}{V(\omega)} \int_{\omega} a_{\mathrm{m}} d V,
$$

where the subscript $m$ refers to the micro-scale, the subscript $M$ refers to the homogenized value, $\langle\bullet\rangle$ is the volume average, and $V(\omega)$ is the volume of the meso-scale volume element $\omega$. In particular, the macro-stress tensor $\boldsymbol{\sigma}_{\mathrm{M}}$ and the macro-strain tensor $\varepsilon_{\mathrm{M}}$ are defined as

$$
\begin{aligned}
& \boldsymbol{\sigma}_{\mathrm{M}}=\left\langle\boldsymbol{\sigma}_{\mathrm{m}}\right\rangle=\frac{1}{V(\omega)} \int_{\omega} \boldsymbol{\sigma}_{\mathrm{m}} d V, \\
& \varepsilon_{\mathrm{M}}=\left\langle\varepsilon_{\mathrm{m}}\right\rangle=\frac{1}{V(\omega)} \int_{\omega} \varepsilon_{\mathrm{m}} d V .
\end{aligned}
$$

In the elastic regime, the micro-stress tensor is related to the micro-strain tensor through the material fourth-order tensor $\mathbb{C}_{\mathrm{m}}$ with

$$
\sigma_{\mathrm{m}}=\mathbb{C}_{\mathrm{m}}: \varepsilon_{\mathrm{m}} .
$$

When performing the homogenization, the relative size of the meso-scale volume element with respect to the micro-structure size is of prime importance. If the volume-element on which the averaging is performed is large enough, it is called Representative Volume Element (RVE). To be considered as a RVE, the volume-element should be statistically representative so that it is entirely typical of the whole mixture on average [21]. Moreover the volume element is representative when the effective constitutive response is independent with respect to the "energetically consistent" boundary conditions, as it will be discussed later.

Assuming $\omega$ is an RVE, a unique effective material tensor $\mathbb{C}_{\mathrm{M}}^{\mathrm{eff}}$ can be defined such that

$$
\sigma_{\mathrm{M}}=\mathbb{C}_{\mathrm{M}}^{\mathrm{eff}}: \varepsilon_{\mathrm{M}},
$$

for any RVE $\omega$ [40]. Defining as $a_{\mathrm{m}}^{\prime}$ the perturbation of the micro-scale field $a_{\mathrm{m}}$ 
around its average value $\left\langle a_{\mathrm{m}}\right\rangle$, and combining Eqs. (7-10) lead to

$$
\begin{array}{r}
\mathbb{C}_{\mathrm{M}}^{\mathrm{eff}}: \varepsilon_{\mathrm{M}}=\boldsymbol{\sigma}_{\mathrm{M}}=\frac{1}{V(\omega)} \int_{\omega}\left(\left\langle\mathbb{C}_{\mathrm{m}}\right\rangle+\mathbb{C}_{\mathrm{m}}^{\prime}\right):\left(\left\langle\varepsilon_{\mathrm{m}}\right\rangle+\varepsilon_{\mathrm{m}}^{\prime}\right) d V= \\
\left\langle\mathbb{C}_{\mathrm{m}}\right\rangle: \varepsilon_{\mathrm{M}}+\left\langle\mathbb{C}_{\mathrm{m}}^{\prime}: \varepsilon_{\mathrm{m}}^{\prime}\right\rangle,
\end{array}
$$

which shows that the effective material tensor is not equal to the meso-scale volume average of the micro-scale material tensor $\left\langle\mathbb{C}_{\mathrm{m}}\right\rangle$. This last expression corresponds to the Voigt upper bound $\overline{\mathbb{C}}_{M}$ of the effective material tensor. In all generalities, an upper (lower) bound $\mathbb{C}_{U}\left(\mathbb{C}_{\mathrm{L}}\right)$ of the tensor $\mathbb{C}$ is defined such that $\varepsilon:\left(\mathbb{C}_{\mathrm{U}}-\mathbb{C}\right): \varepsilon \geqslant 0\left(\varepsilon:\left(\mathbb{C}-\mathbb{C}_{\mathrm{L}}\right): \varepsilon \geqslant 0\right)$ for any non-zero deformation tensor $\varepsilon$, and we use the notation $\mathbb{C}_{U} \geqslant \mathbb{C} \geqslant \mathbb{C}_{\mathrm{L}}$. Applying the same relations by considering the compliance tensor $\mathbb{S}$ leads to a similar conclusion: the effective compliance tensor $\mathbb{S}_{\mathrm{M}}^{\mathrm{eff}}$ such that

$$
\varepsilon_{\mathrm{M}}=\mathbb{S}_{\mathrm{M}}^{\mathrm{eff}}: \sigma_{\mathrm{M}},
$$

is different from the meso-scale volume average of the micro-scale compliance tensor $\left\langle\mathbb{S}_{\mathrm{m}}\right\rangle$. The inverse of this last expression corresponds to the Reuss lower bound $\mathbb{C}_{M}$ of the effective material tensor.

To be energetically consistent, the effective tensor $\mathbb{C}_{\mathrm{M}}^{\text {eff }}$ should satisfy the Hill-Mandel principle, which implies the equality of the internal energy at both scales, i.e.

$$
\sigma_{\mathrm{M}}: \varepsilon_{\mathrm{M}}=\frac{1}{V(\omega)} \int_{\omega} \sigma_{\mathrm{m}}: \varepsilon_{\mathrm{m}} d V .
$$

Using Eqs. (7-10), this equation reduces to

$$
\begin{array}{r}
\varepsilon_{\mathrm{M}}: \mathbb{C}_{\mathrm{M}}^{\mathrm{eff}}: \varepsilon_{\mathrm{M}}=\frac{1}{V(\omega)} \int_{\omega} \boldsymbol{\sigma}_{\mathrm{m}}: \varepsilon_{\mathrm{m}} d V= \\
\frac{1}{V(\omega)} \int_{\omega}\left(\left\langle\boldsymbol{\sigma}_{\mathrm{m}}\right\rangle+\boldsymbol{\sigma}_{\mathrm{m}}^{\prime}\right):\left(\left\langle\varepsilon_{\mathrm{m}}\right\rangle+\varepsilon_{\mathrm{m}}^{\prime}\right) d V=\varepsilon_{\mathrm{M}}: \mathbb{C}_{\mathrm{M}}^{\mathrm{eff}}: \varepsilon_{\mathrm{M}}+\left\langle\boldsymbol{\sigma}_{\mathrm{m}}^{\prime}: \varepsilon_{\mathrm{m}}^{\prime}\right\rangle .
\end{array}
$$

Therefore the resolution of the meso-scale boundary value problem should satisfy

$$
\int_{\omega} \boldsymbol{\sigma}_{\mathrm{m}}^{\prime}: \varepsilon_{\mathrm{m}}^{\prime} d V=0
$$

This condition is satisfied for the Voigt and Reuss assumptions, which respectively state a constant strain field, i.e. $\varepsilon_{\mathrm{m}}^{\prime}=0$, and a constant stress field, i.e. $\boldsymbol{\sigma}_{\mathrm{m}}^{\prime}=0$, which lead to the upper and lower bounds of the effective tensor, respectively.

In order to estimate the effective material tensor $\mathbb{C}_{\mathrm{M}}^{\text {eff }}$ from the resolution of the meso-scale BVP, boundary conditions should be applied on the RVE $\omega$. The Hill-Mandell condition (15) can be rewritten $[16,17,41]$ in the absence of body forces as

$$
0=\int_{\partial \omega}\left(\boldsymbol{t}_{\mathrm{m}}-\boldsymbol{\sigma}_{\mathrm{M}} \cdot \boldsymbol{n}_{\mathrm{m}}\right) \cdot\left(\boldsymbol{u}_{\mathrm{m}}-\boldsymbol{\varepsilon}_{\mathrm{M}} \cdot \boldsymbol{x}\right) d S,
$$


where $\partial \omega$ is the boundary of the meso-scale volume $\omega, S$ is its surface, $\boldsymbol{n}_{\mathrm{m}}$ is its outward unit normal, $\boldsymbol{x}$ is the position vector, $\boldsymbol{t}_{\mathrm{m}}=\boldsymbol{\sigma}_{\mathrm{m}} \cdot \boldsymbol{n}_{\mathrm{m}}$ is the surface traction, and where $\boldsymbol{u}_{\mathrm{m}}$ is the micro-displacement field. To define "energetically consistent" boundary conditions, the displacement field $\boldsymbol{u}_{\mathrm{m}}$ is decomposed into an average value $\left\langle\boldsymbol{u}_{\mathrm{m}}\right\rangle=\varepsilon_{\mathrm{M}} \cdot \boldsymbol{x}$ and into a fluctuation field $\boldsymbol{u}_{\mathrm{m}}^{\prime}$ so that the Hill-Mandel principle (16) is finally rewritten [41] as

$$
\int_{\partial \omega} \boldsymbol{t}_{\mathrm{m}} \cdot \boldsymbol{u}_{\mathrm{m}}^{\prime} d S=0
$$

The four main BCs satisfying this equation are

- The Kinematic Uniform Boundary Conditions (KUBCs) for which there is no fluctuation on the boundary, i.e.

$$
\boldsymbol{u}_{\mathrm{m}}^{\prime}=0 \text { on } \partial \omega
$$

- The Static Uniform Boundary Conditions (SUBCs) for which $\boldsymbol{t}_{\mathrm{m}}=\boldsymbol{\sigma}_{\mathrm{M}} \cdot \boldsymbol{n}_{\mathrm{m}}$ on $\partial \omega$; In the case of parallelepiped RVEs for which the boundary can be separated in opposite faces $\partial \omega^{-}$and $\partial \omega^{+}$, this also corresponds to the minimal kinematic boundary conditions [17], i.e.

$$
\int_{\partial \omega^{ \pm}}\left(\boldsymbol{u}_{\mathrm{m}}^{\prime} \otimes \boldsymbol{n}_{\mathrm{m}}\right) d S=0
$$

- The Orthogonal Uniform Mixed Boundary Conditions (OUMBCs), for which a combination of constrained displacements in one direction and surface tractions in the other directions is used ${ }^{1}$;

- The Periodic Boundary Conditions (PBCs) for which one has on the opposite faces

$$
\boldsymbol{u}_{\mathrm{m}}^{\prime}\left(\boldsymbol{x}^{+}\right)=\boldsymbol{u}_{\mathrm{m}}^{\prime}\left(\boldsymbol{x}^{-}\right) \forall \boldsymbol{x}^{+} \in \partial \omega^{+} \text {and corresponding } \boldsymbol{x}^{-} \in \partial \omega^{-},
$$

with $\int_{\partial \omega^{ \pm}} \boldsymbol{u}_{\mathrm{m}}^{\prime} d S=0$ to remove the rigid mode motion.

As previously stated, if the meso-scale volume-element $\omega$ on which the averaging is performed is large enough, the so-called RVE is statistically representative and a unique material tensor $\mathbb{C}_{\mathrm{M}}^{\mathrm{eff}}$ can be obtained for these different

\footnotetext{
${ }^{1}$ Although not true for general MBCs, particular MBCs such as the orthogonal uniform ones can be defined in a particular way as to satisfy the Hill-Mandel condition (17), see the discussion in [42]. Assuming a rectangular parallelepiped RVE, on every face we constrain along one direction -says $x$ - the displacements to $u_{\mathrm{m} x}=\sum_{i=x}^{z} \varepsilon_{\mathrm{M} x i} x_{i}$, so that $u_{\mathrm{m} x}^{\prime}=0$, and along the two other directions $t_{\mathrm{m} j}=\sum_{k=x}^{z} \sigma_{\mathrm{M} j k} n_{\mathrm{m} k}, j=y, z$. As a result, since $u_{\mathrm{m} x}^{\prime}=0$, the Hill-Mandel Eq. (17) is rewritten $\sum_{j=y}^{z} \sum_{k=x}^{z} \sigma_{\mathrm{M}_{j k}} \int_{\partial \omega^{ \pm}} n_{\mathrm{m} k} \boldsymbol{u}_{\mathrm{m} j}^{\prime} d S$, which vanishes by constraining $\int_{\partial \omega^{ \pm}} n_{\mathrm{m} k} \boldsymbol{u}_{\mathrm{m} j}^{\prime} d S=0$ for $j=y, z$ and $k=x, y, z$.
} 
"energetically consistent" boundary conditions. If the volume element is not large enough the homogenization provides an apparent material tensors $\mathbb{C}_{\mathrm{M}}$, which depends on the applied boundary conditions, but also on the particular realization of the micro-structure considered. In this case, the meso-scale volume-element $\omega$ is called Statistical Volume Element (SVE) [22].

Therefore the material tensors obtained using SVEs face two sources of uncertainties: one contribution resulting from the applied boundary conditions and the other one from the uncertainties in the material micro-structure. However as it will be seen in Section 3.4, the uncertainties resulting from the microstructure randomness, the grain distribution and orientation, is more important than the ones resulting from the applied BCs in the case of the studied polycrystalline material. This paper will only consider OUMBCs to estimate the apparent meso-scale material tensors $\mathbb{C}_{\mathrm{M}}$.

In the next section we discuss how this apparent material tensor $\mathbb{C}_{M}$ is computed in the computational homogenization framework before detailing the process to generate the different SVEs.

\subsection{Evaluation of the apparent meso-scale material tensor}

Computing the apparent meso-scale material tensor from the finite element resolution of a meso-scale volume-element $\omega$ can be done in different ways. In [32] and [33], it was achieved with the help of a minimization procedure and Huet's partition theorem [40]. It can also be estimated directly from the stiffness matrix of the FE model following the developments in $[16,17]$. This last method is adopted herein.

In the absence of body forces, the macro-stress tensor (7) can be rewritten

$$
\boldsymbol{\sigma}_{\mathrm{M}}=\frac{1}{V(\omega)} \int_{\partial \omega} \boldsymbol{t}_{\mathrm{m}} \otimes \boldsymbol{x} d S
$$

When considering a finite element discretization of the SVE and when applying one of the energetically consistent boundary conditions (18-20), there are $N_{\text {nd }}$ nodes with prescribed displacements on the boundary $\partial \omega, N_{\text {nd }}$ depending on the type of boundary conditions. Let $\boldsymbol{x}^{p}$ be the position vector of these nodes. The discretized form of Eq. (21) thus reads

$$
\boldsymbol{\sigma}_{\mathrm{M}}=\frac{1}{V(\omega)} \sum_{p=1}^{N_{\mathrm{nd}}} \boldsymbol{f}^{p} \otimes \boldsymbol{x}^{p},
$$

where $\boldsymbol{f}^{p}$ corresponds to the resulting external nodal forces at the prescribed nodes. In linear elasticity, the equilibrium between external and internal forces can be written as

$$
\sum_{q=1}^{N_{\mathrm{nd}}} \boldsymbol{K}_{\mathrm{M}}^{p q} \cdot \boldsymbol{u}^{q}=\boldsymbol{f}^{p},
$$

where $p$ and $q$ correspond to the different $N_{\text {nd }}$ prescribed nodes, and where $\boldsymbol{K}_{\mathrm{M}}^{p q}$ is the stiffness tensor at nodes $p$ and $q$ obtained thanks to the condensation of 


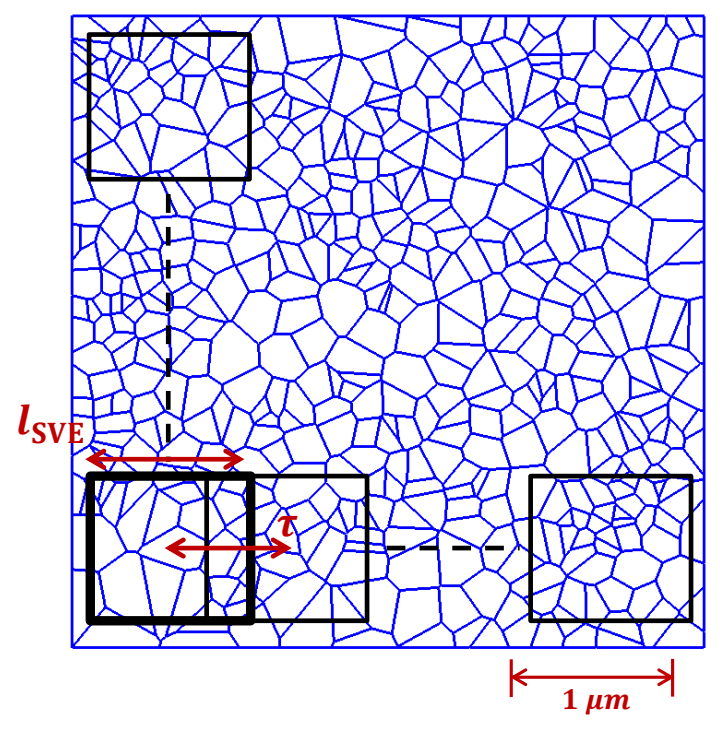

Figure 4: SVEs generation strategy

the internal nodes [17]. This condensation depends on the kind of boundary conditions considered, see [17] for details. Substituting Eq. (23) in Eq. (22) results in

$$
\boldsymbol{\sigma}_{\mathrm{M}}=\frac{1}{V(\omega)} \sum_{p=1}^{N_{\mathrm{nd}}} \sum_{q=1}^{N_{\mathrm{nd}}}\left(\boldsymbol{K}_{\mathrm{M}}^{p q} \cdot \boldsymbol{u}^{q}\right) \otimes \boldsymbol{x}^{p},
$$

or again, according to the definition of the deformation tensor

$$
\boldsymbol{\sigma}_{\mathrm{M}}=\frac{1}{V(\omega)} \sum_{p=1}^{N_{\mathrm{nd}}} \sum_{q=1}^{N_{\mathrm{nd}}}\left(\boldsymbol{x}^{p} \otimes \boldsymbol{K}_{\mathrm{M}}^{p q} \otimes \boldsymbol{x}^{q}\right): \varepsilon_{\mathrm{M}} .
$$

The apparent elasticity tensor $\mathbb{C}_{\mathrm{M}}$ is then directly obtained as

$$
\mathbb{C}_{\mathrm{M}}=\frac{1}{V(\omega)} \sum_{p=1}^{N_{\mathrm{nd}}} \sum_{q=1}^{N_{\mathrm{nd}}} \boldsymbol{x}^{p} \otimes \boldsymbol{K}_{\mathrm{M}}^{p q} \otimes \boldsymbol{x}^{q} .
$$

With a view to the generation of the material tensor random field, the fourth order symmetric elasticity tensor $\mathbb{C}_{M}$ is represented using the Voigt or Kelvin notation. Out of the 81 components of the tensor $\mathbb{C}_{\mathrm{M}}$, only 21 components are enough to fully characterized the elastic operator, which can be represented by a $6 \times 6$ symmetric elasticity matrix $\boldsymbol{C}_{\mathrm{M}}$.

\subsection{SVEs generations}

Using Eq. (26), we can extract the apparent material tensor $\mathbb{C}_{M}$, or its matrix version $\boldsymbol{C}_{\mathrm{M}}$, from a finite element discretization of an SVE. An example 
of SVE is illustrated in Fig. 3. The purpose of this work is to evaluate the random field of the material tensor at the meso-scale, which requires to consider several SVE realizations.

To this end, the following procedure is considered in the generation of the SVEs. Poisson-Voronoï tessellations are generated as detailled in Appendix A to represent a columnar polycrystalline material obtained by low pressure chemical vapor deposition (LPCVD). Each grain possesses a random orientation. One tessellation is illustrated in Fig. 4. For a given SVE size, several SVEs of the same size can be extracted from a tessellation following the moving window technique [6] illustrated in Fig. 4. Toward this end, an initial SVE is extracted from the tessellation - using the methodology reported in Appendix A. A series of other SVEs, whose centers are separated by a vector $\tau$ from the initial SVE center, can then be extracted, see Fig. 4. Note that due to the statistical isotropic nature of the Poisson-Voronoï tessellation, considering vectors $\tau$ along one direction is enough. A sufficient number of large Poisson-Voronoï tessellations are used to obtain a high number of SVEs in order for the description of the random field to converge. As we assume a homogeneous meso-scale random field in this work, a set of tessellation realizations -such a realization is illustrated in Fig. 4- is enough to characterize the meso-scale random field of the whole macro-structure. The size of each tessellation is constrained by the moving window technique: the tessellation should be large enough in order to be able to capture the spatial correlations, i.e. the size should be large enough for all the spatial correlations to reach zero when moving the window. The size of the tesselations is thus not related to the size of the macro-structure in the case of a homogeneous random field. In the case of inhomogeneous random fields, more efforts are required to describe the spatial variation of the random field, and it is out of the scope of the present work.

The spatial cross-correlation matrix $\boldsymbol{R}_{\boldsymbol{C}_{\mathrm{M}}}(\boldsymbol{\tau})$, of the assumed homogeneous field $\boldsymbol{C}_{\mathrm{M}}(\boldsymbol{x})$ of the apparent effective tensor, is evaluated as follows

$$
\begin{array}{r}
R_{\boldsymbol{C}_{\mathrm{M}}}^{(r s)}(\boldsymbol{\tau})=\frac{\mathbb{E}\left[\left(C_{\mathrm{M}}^{(r)}(\boldsymbol{x})-\mathbb{E}\left[C_{\mathrm{M}}^{(r)}\right]\right)\left(C_{\mathrm{M}}^{(s)}(\boldsymbol{x}+\boldsymbol{\tau})-\mathbb{E}\left[C_{\mathrm{M}}^{(s)}\right]\right)\right]}{\sigma_{{ }_{\mathrm{M}}^{(r)}} \sigma_{C_{\mathrm{M}}^{(s)}}} \quad \forall r, s=1, \ldots, 21,
\end{array}
$$

where $C_{\mathrm{M}}^{(r)}(\boldsymbol{x})$ is the $r^{\text {th }}$ element out of the 21 relevant elements of the material tensor $\boldsymbol{C}_{\mathrm{M}}$ evaluated at position $\boldsymbol{x}, \mathbb{E}$ is the expectation operator, and where $\sigma_{C_{\mathrm{M}}^{(r)}}=\sqrt{\mathbb{E}\left[\left(C_{\mathrm{M}}^{(r)}-\mathbb{E}\left[C_{\mathrm{M}}^{(r)}\right]\right)^{2}\right]}$ is the standard deviation of $C_{\mathrm{M}}^{(r)}$.

\subsection{Application to the poly-silicon case}

With a view toward the study of a MEMS resonator, we consider micro-size beams made of silicon organized in a polycrystalline structure. Silicon is one of the most common material present in MEMS. The uncertainties are coming from two sources: the grain size/geometry and the grain orientations. 
The first one is captured by the Voronoï tessellation. Accordingly to the typical fabrication process of the considered poly-silicon MEMS by low pressure chemical vapor deposition (LPCVD) at $580^{\circ} \mathrm{C}$, for a thickness of $2 \mu \mathrm{m}$, an average grain diameter $\bar{d}$ of about $200 \mathrm{~nm}$ is adopted, yielding an average area of grains $\bar{a}=\pi \bar{d}^{2} / 4$. One tessellation is illustrated in Fig. 4. Moreover, since the considered MEMS structures are fabricated through LPCVD, similar micro material structures (at the grain scale) will be obtained on the whole MEMSstructure, which justifies the use of the homogeneous random field.

The second one is represented by assigning random orientations to the grains. Indeed, Silicon material is anisotropic, with a cubic symmetry, and the properties of a Silicon grain depend on its orientation with respect to the crystal lattice. The material properties of a silicon crystal are studied in [43]. Its Young's modulus can range from $130 \mathrm{GPa}$ up to $188 \mathrm{GPa}$ and its Poisson ratio can range from 0.048 up to 0.4. For the silicon crystal oriented with [100], [010] and [001] along the Cartesian coordinates, the crystal elasticity tensor $\boldsymbol{C}_{\mathrm{S}}$ -where the subscript $\mathrm{S}$ indicates the properties for the single crystal- reads, in GPa,

$$
\boldsymbol{C}_{\mathrm{S}}=\left[\begin{array}{cccccc}
165.7 & 63.9 & 63.9 & 0 & 0 & 0 \\
63.9 & 165.7 & 63.9 & 0 & 0 & 0 \\
63.9 & 63.9 & 165.7 & 0 & 0 & 0 \\
0 & 0 & 0 & 79.6 & 0 & 0 \\
0 & 0 & 0 & 0 & 79.6 & 0 \\
0 & 0 & 0 & 0 & 0 & 79.6
\end{array}\right]
$$

In order to apply the 3-scale stochastic method in Section 5, we study 3D SVEs representing the poly-silicon structure. As the micro-structure is columnar, the material properties are constant along the thickness of the SVE and a first-order computational homogenization scheme is applied. For more complex micro-structures, second-order boundary conditions should be considered as in [44]. As the width (thickness) of the macro-scale beam is small compared to its length, 1D-finite elements will be considered at the macro-scale and the SVE width (thickness) considered is the one of the macro-beam. Note that the thickness of the SVE should not influence the elasticity tensor as the material properties are constant along the thickness. Therefore both the width and the thickness of the macro-beam are implicitly considered in the 3D homogenization and, in turn, in the beam discretization. The only relevant geometric parameter to study the size effect of the SVE is thus its length. This also means that the spatial correlation is only required in one direction. We consider four different SVE lengths, $l_{\mathrm{SVE}}$, successively equal to $0.1 \mu \mathrm{m}, 0.2 \mu \mathrm{m}, 0.4 \mu \mathrm{m}$, and $0.6 \mu \mathrm{m}$. The width and height of the SVEs are respectively $0.5 \mu \mathrm{m}$ and $0.1 \mu \mathrm{m}$.

Applying the described homogenization methodology, the meso-scale mechanical properties distribution can be obtained through the evaluation of the material tensor $\boldsymbol{C}_{\mathrm{M}}$ using Eq. (26) for the different SVE realizations. The number of tessellations generated are 1084, 855, 312, and 117 for respectively an SVE length $l_{\mathrm{SVE}}$ of $0.1,0.2,0.4$, and $0.6 \mu \mathrm{m}$. The distance between the centers of thesuccessive SVEs extracted from the tessellation is $0.5 \times l_{\mathrm{SVE}}$ for a $l_{\mathrm{SVE}}$ of 


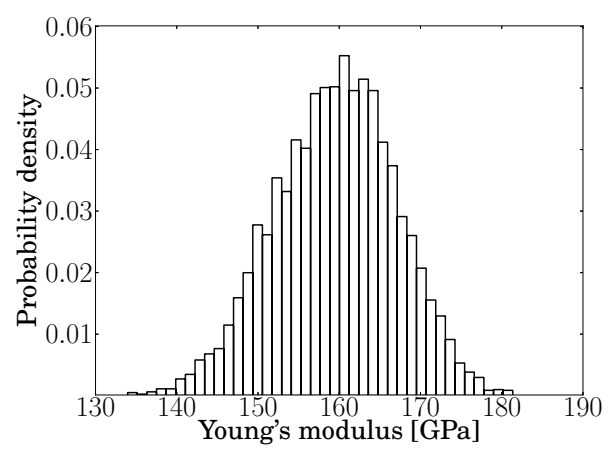

Figure 5: Samples of Young's modulus obtained for an SVE length of $0.2 \mu \mathrm{m}$

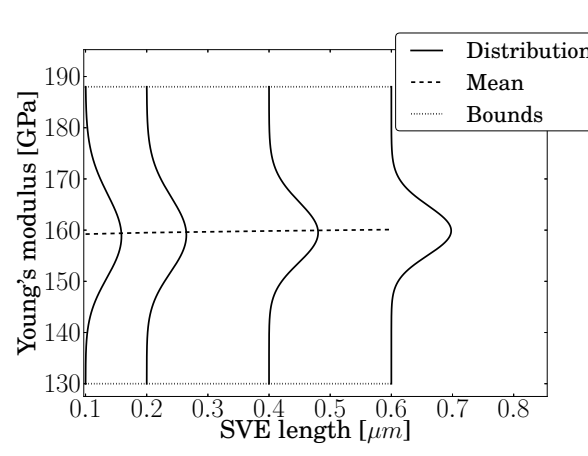

(a) Evolution of the Young's modulus

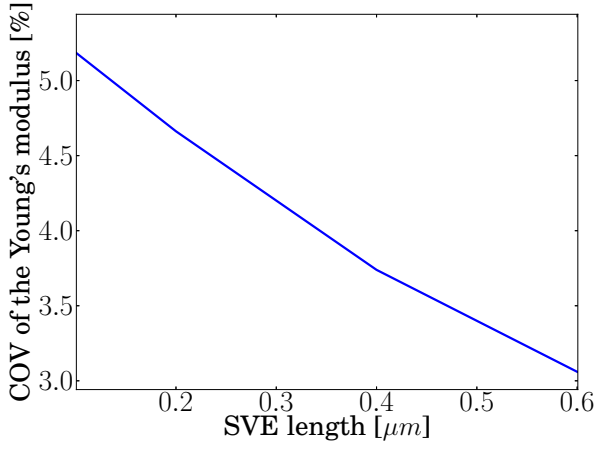

(b) Evolution of the variance

Figure 6: Meso-scale Young's modulus $E_{x}$ distribution for different SVE lengths ( $x$ is along the SVE length)

0.1 and $0.2 \mu \mathrm{m}$ and $0.25 \times l_{\mathrm{SVE}}$ for the 2 remaining SVE lengths. As an example the resulting histogram of the Young's modulus $E_{x}{ }^{2}$ along the $x$-direction ( $x$ is along the SVE length, $y$ along the width, and $z$ along its height) is illustrated in Fig. 5 for an SVE of length $l_{\mathrm{SVE}}=0.2 \mu \mathrm{m}$. The effect of the SVE length on the meso-scale properties is depicted in Fig. 6. Figure 6(a) represents the evolution of the distribution of the Young's modulus for different SVE lengths $l_{\mathrm{SVE}}$. The silicon bounds are also reported on that figure. One can see in the figure that a larger SVE involves a more peaky distribution. The mean Young's modulus is found to be around $160 \mathrm{GPa}$. The evolution of the Coefficient of Variation (COVs) of the meso-scale Young's modulus, $\mathrm{COV}=\frac{\sigma_{E_{x}}}{\mathbb{E}\left[E_{x}\right]} \times 100 \%$, with $\sigma_{E_{x}}$ the standard deviation of the meso-scale Young's modulus along the beam axis and $\mathbb{E}\left[E_{x}\right]$ its expectation, with respect to the SVE length is reported in Fig. 6(b). As expected, the coefficient of variation decreases with the SVE length.

\footnotetext{
${ }^{2}$ For conciseness, in what follows we drop the subscript $M$ which refers to the homogenized meso-scale properties.
} 


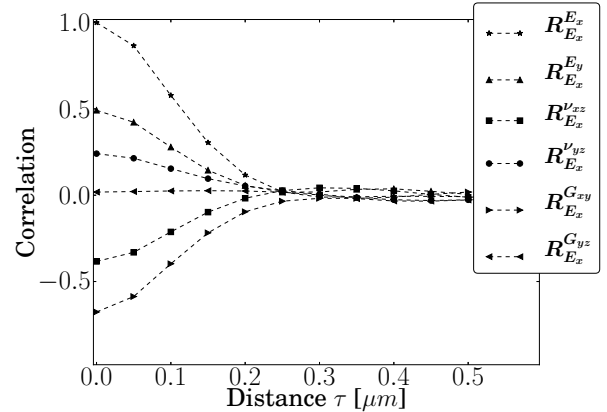

(a) Cross-correlations of $E_{x}$

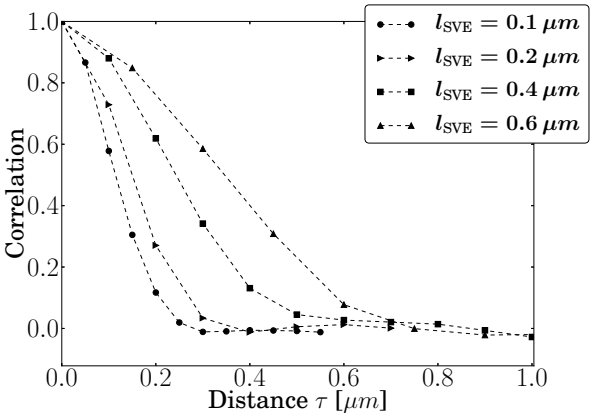

(b) Auto-correlation of $E_{x}$

Figure 7: Two-points statistics for different values of the distance $\tau$ along $x$ between the centers of the SVEs. (a) Auto- and cross-correlations of the Young's modulus in the $x$ direction with respect to different material properties for an SVE length $l_{\mathrm{SVE}}=0.1 \mu \mathrm{m}(x$ is along the SVE length, $y$ along the width, and $z$ along its height). (b) The auto-correlation of the Young's modulus for different SVE lengths.

The auto-correlation of the Young's modulus $E_{x}$ and the cross-correlations of the Young's modulus $E_{x}$, with other material properties extracted from the elasticity tensor, are computed for an SVE length of $l_{\mathrm{SVE}}=0.1 \mu \mathrm{m}$ using Eq. (27) and are reported in Fig. 7(a) for different values of the distance $\tau$-along $x$. Such an analysis was previously achieved in [45] in the case of a Bernoulli lattice. The evolution with the distance $\tau$-along $x$ - of the auto-correlation of the Young's modulus $E_{x}$ is also evaluated using Eq. (27) and is illustrated in Fig. 7(b) for the four considered SVE lengths.

The following conclusions can be drawn from Fig. 7:

- As expected for $\tau=0$, the auto-correlation is equal to the unity and the cross-correlations are always $<1$ and $>-1$;

- The correlations obtained for $\tau=l_{\mathrm{SVE}}$, i.e. when the two SVEs are adjacent, are low but do not vanish as two adjacent SVEs share some common grains; This effect decreases with the increase of the SVE length, as the proportion of shared grains also decreases; As an example, the autocorrelation obtained for $\tau=l_{\mathrm{SVE}}$ is $\approx 0.6$ for an SVE length of $0.1 \mu \mathrm{m}$ and is $\approx 0.17$ for an SVE of $0.4 \mu \mathrm{m}$, see Fig. $7(\mathrm{~b})$;

- For longer meso-scale volume elements, sharing of common grains takes place at longer distances between SVEs and the correlation lengths defined by Eq. (4) of the Young's modulus for the different SVE lengths increases, see Tab. 1;

- When $\tau$ increases the auto- and cross-correlations decreases to 0 as does the probability to share some grains;

- The cross-correlation between $E_{x}$ and $E_{y}$ is positive while the crosscorrelation between $E_{x}$ and the Poisson ratio $\nu_{x z}$ and the in-plane shear 
Table 1: Correlation length $l_{E_{x}}$ of the Young's modulus $E_{x}$ for different SVE lengths

\begin{tabular}{|c|c|}
\hline SVE length $[\mu \mathrm{m}]$ & Correlation Length $[\mu \mathrm{m}]$ \\
\hline 0.1 & 0.233 \\
\hline 0.2 & 0.307 \\
\hline 0.4 & 0.504 \\
\hline 0.6 & 0.693 \\
\hline
\end{tabular}

modulus $G_{x y}$ is negative because of the cubic symmetry of the Silicon crystal; Indeed the Silicon crystal has a minimum Young's modulus along

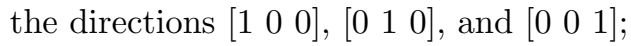

- The cross-correlation between $E_{x}$ and the out-of-plane shear modulus is almost zero.

Finally, the effect of the applied BCs on the SVE homogenization results is investigated. The standard deviations of the Young's modulus along $x$ obtained for KUBCs, OUMBCs, and SUBCs are reported in Tab. 2. Three different SVE lengths are successively considered: $0.1 \mu \mathrm{m}, 0.2 \mu \mathrm{m}$, and $0.4 \mu \mathrm{m}$. The difference in the meso-scale distribution standard deviation obtained with the OUMBCs distribution is found to be lower than $2 \%$ as compared with the other BCs, justifying the use of the sole OUMBCs.

Table 2: Standard deviation of the Young's modulus $E_{x}$ obtained with different boundary conditions and SVE lengths

\begin{tabular}{|c||c|c|c|}
\hline SVE lengths $[\mu \mathrm{m}]$ & KUBC & OUMBC & SUBC \\
\hline 0.1 & $\sigma_{E_{x}}=8.33 \mathrm{GPa}$ & $\sigma_{E_{x}}=8.43 \mathrm{GPa}$ & $\sigma_{E_{x}}=8.43 \mathrm{GPa}$ \\
\hline 0.2 & $\sigma_{E_{x}}=7.22 \mathrm{GPa}$ & $\sigma_{E_{x}}=7.31 \mathrm{GPa}$ & $\sigma_{E_{x}}=7.31 \mathrm{GPa}$ \\
\hline 0.4 & $\sigma_{E_{x}}=6.02 \mathrm{GPa}$ & $\sigma_{E_{x}}=6.11 \mathrm{GPa}$ & $\sigma_{E_{x}}=6.13 \mathrm{GPa}$ \\
\hline
\end{tabular}

\section{Mesoscopic scale: elasticity tensor generation}

In order to obtain the meso-scale random field for the macro-scale SFEM analyzes, a generator is defined using the realizations of the micro-meso homogenization as inputs. With such a method it becomes straightforward and computationally efficient to study different structural probabilistic problems as the generator provides as many realizations as required.

In order for the random field generator to lead to physically meaningful random elasticity tensors, an absolute lower bound $\boldsymbol{C}_{\mathrm{L}}$ is enforced ${ }^{3}$. This enforcement of the absolute lower bound also ensures the existence of the expectation of the generated elasticity tensor inverses.

\footnotetext{
${ }^{3}$ The lower (upper) bound $\boldsymbol{C}_{\mathrm{L}}\left(\boldsymbol{C}_{\mathrm{U}}\right)$ of a matrix $\boldsymbol{C}$ is defined in a similar way as for the fourth order tensors in Section 3.1, i.e. such that $\boldsymbol{\varepsilon}^{T}\left(\boldsymbol{C}-\boldsymbol{C}_{\mathrm{L}}\right) \boldsymbol{\varepsilon} \geqslant 0\left(\boldsymbol{\varepsilon}^{T}\left(\boldsymbol{C}_{\mathrm{U}}-\boldsymbol{C}\right) \boldsymbol{\varepsilon} \geqslant 0\right)$ for any non-zero vector $\varepsilon \in \mathbb{R}^{6}$ with Voigt notations, and we use the notation $\boldsymbol{C}_{\mathrm{U}} \geqslant \boldsymbol{C} \geqslant \boldsymbol{C}_{\mathrm{L}}$
} 
In this section, after having defined an appropriate lower bound for the polycrystalline materials, the generation of the meso-scale random field is detailed. Finally, in the case of the poly-silicon material, the generated meso-scale random fields properties are compared to the original distributions obtained from the SVE resolutions presented in Section 3.4.

\subsection{Definition of the absolute lower bound}

The absolute lower bound is defined so that $\boldsymbol{C}_{\mathrm{M}}-\boldsymbol{C}_{\mathrm{L}}$ is a positive semidefinite matrix for any generated elasticity tensor $\boldsymbol{C}_{\mathrm{M}}$, and we use the notation $\boldsymbol{C}_{\mathrm{M}} \geqslant \boldsymbol{C}_{\mathrm{L}}$. Although only the lower bound will be enforced when generating the random field, both the lower and upper bounds are discussed hereafter for the sake of generality.

As discussed in Section 3.1, for heterogeneous materials made of different material phases, there are two absolute bounds which are used to define the variation range of the elasticity tensor on a representative volume element (RVE): the upper bound -or Voigt bound- $\overline{\boldsymbol{C}}_{\mathrm{M}}$ and the lower bound -or Reuss bound$\underline{C}_{\mathrm{M}}$. In the case of a multi-phase material, these bounds read

$$
\begin{aligned}
& \overline{\boldsymbol{C}}_{\mathrm{M}}=\left\langle\boldsymbol{C}_{\mathrm{m}}\right\rangle=\sum_{i=1}^{n} f_{i} \boldsymbol{C}_{i} \\
& \underline{\boldsymbol{C}}_{\mathrm{M}}=\left(\left\langle\boldsymbol{C}_{\mathrm{m}}^{-1}\right\rangle\right)^{-1}=\left(\sum_{i=1}^{n} f_{i} \boldsymbol{C}_{i}^{-1}\right)^{-1},
\end{aligned}
$$

respectively, where $\boldsymbol{C}_{i}(i=1,2, \ldots, n)$ is the elasticity tensor of phase $i$ and $f_{i}$ the volume fraction of phase $i$, which satisfies $\sum_{i=1}^{n} f_{i}=1$.

However, when considering different SVEs, the bounds (29-30) cannot be determined anymore, as the volume fractions $f_{i}$ of each phase is different for each SVE. Moreover, in the problem of a polycrystalline material, which is an aggregate of grains based on the same crystal but with random orientations, as the same anisotropic material is considered in each grain, of random shape and orientation, it is not possible to define $\boldsymbol{C}_{i}$ and $f_{i}$, as the different elasticity tensors $\boldsymbol{C}_{i}$ are expressions of the same tensor in different coordinates systems. For the same reasons, it is not possible to defined bounds, which satisfy $C_{\mathrm{L}} \leqslant$ $\boldsymbol{C}_{\mathrm{M}} \leqslant \boldsymbol{C}_{\mathrm{U}}$, directly from the grains material tensors $\boldsymbol{C}_{i}$ as

$$
\begin{aligned}
& \boldsymbol{C}_{\mathrm{U}}=\max \left\{\boldsymbol{C}_{i} \mid i=1,2, \ldots, n\right\} \\
& \boldsymbol{C}_{\mathrm{L}}=\min \left\{\boldsymbol{C}_{i} \mid i=1,2, \ldots, n\right\} .
\end{aligned}
$$

Indeed, as all the grains are associated to elasticity tensors $\boldsymbol{C}_{i}(i=1,2, \ldots, n)$ which are the same tensors in different coordinates, they have the same eigenvalues.

In this work we propose an efficient method to define the lower bound $\boldsymbol{C}_{\mathrm{L}}$ and the upper bound $\boldsymbol{C}_{\mathrm{U}}$ of a polycrystalline material, for any size of the SVEs. As we have described previously, $\boldsymbol{C}_{i}(i=1,2, \ldots, n)$ are the expressions of the same elasticity tensor in different coordinates. Let $\boldsymbol{C}_{\mathrm{S}}$ be the elasticity tensor 
of a single crystal. To define the two bounds $\boldsymbol{C}_{\mathrm{L}}$ and $\boldsymbol{C}_{\mathrm{U}}$, we need to guaranty that $\boldsymbol{C}_{\mathrm{L}} \leqslant \boldsymbol{C}_{\mathrm{S}} \leqslant \boldsymbol{C}_{\mathrm{U}}$ for any orientation of the crystal, and thus for any rotation of $\boldsymbol{C}_{\mathrm{S}}$. By satisfying this relation, $\boldsymbol{C}_{\mathrm{L}}$ and $\boldsymbol{C}_{\mathrm{U}}$ are bounds for the polycrystalline material and for the single crystal. Moreover, as the grains can have any orientation, the bounds are defined as isotropic tensors and are thus characterized by two material parameters only. They can thus be expressed as

$$
C^{\text {iso }}=\left[\begin{array}{cccccc}
2 G+\lambda & \lambda & \lambda & 0 & 0 & 0 \\
\lambda & 2 G+\lambda & \lambda & 0 & 0 & 0 \\
\lambda & \lambda & 2 G+\lambda & 0 & 0 & 0 \\
0 & 0 & 0 & G & 0 & 0 \\
0 & 0 & 0 & 0 & G & 0 \\
0 & 0 & 0 & 0 & 0 & G
\end{array}\right]
$$

for example in terms of the shear modulus $G=\frac{E}{2(1+\nu)}$ and Lamé constant $\lambda=\frac{E \nu}{(1+\nu)(1-2 \nu)}$, with Young's modulus $E$ and Poisson ratio $\nu$.

The two isotropic bounds $\boldsymbol{C}_{\mathrm{L}}^{\text {iso }}$ and $\boldsymbol{C}_{\mathrm{U}}^{\text {iso }}$ can thus be obtained by solving two optimization problems, respectively,

$$
\begin{aligned}
& \min _{G, \lambda \in R^{+}}\left\|C^{\text {iso }}-C_{\mathrm{S}}\right\| \quad \text { subject to } \quad C^{\text {iso }} \leqslant C_{\mathrm{S}} \text { and } \\
& \min _{G, \lambda \in R^{+}}\left\|\boldsymbol{C}^{\text {iso }}-\boldsymbol{C}_{\mathrm{S}}\right\| \quad \text { subject to } \boldsymbol{C}^{\text {iso }} \geqslant \boldsymbol{C}_{\mathrm{S}},
\end{aligned}
$$

where $\|*\|$ denotes the Frobenius norm. According to Eq. (31), the resulting tensors $C_{\mathrm{L}}^{\text {iso }}$ and $C_{\mathrm{U}}^{\text {iso }}$ can be used as bounds of the elasticity tensor for a polycrystalline material.

For the silicon crystal oriented with [100], [010] and [001] along the Cartesian coordinates, the crystal elasticity tensor $\boldsymbol{C}_{\mathrm{S}}$ is given in Eq. (28). The lower and upper bounds, respectively obtained from Eqs. (33) and (34), can be expressed in terms of their corresponding Young's modulus $E$ and Poisson ratio $\nu$ as $C^{\text {iso }}(E, \nu)$ following Eq. (32). After solving the optimization problem, the bounds are found to be $C_{\mathrm{L}}^{\text {iso }}(E=130.0 \mathrm{GPa}, \nu=0.278)$ and $C_{\mathrm{U}}^{\text {iso }}(E=187.9 \mathrm{GPa}, \nu=0.181)$. These two values of the Young's modulus, i.e. $130.0 \mathrm{GPa}$ and $187.9 \mathrm{GPa}$, correspond to the lowest and highest values of Young's moduli that a single silicon crystal can reach.

\subsection{Random field generator}

By introducing the lower bound, $\boldsymbol{C}_{\mathrm{L}}$, of the elasticity tensor, the random elasticity tensor can be computed through

$$
\boldsymbol{C}_{\mathrm{M}}=\boldsymbol{C}_{\mathrm{L}}+\Delta \boldsymbol{C},
$$

where $\Delta \boldsymbol{C}$ is a positive semi-definite matrix for SVEs with only one crystal orientation and positive definite matrix for SVEs with more than one grain orientation. The Cholesky decomposition algorithm [8] can be used directly to obtain the positive definite matrix, which is expressed as

$$
\Delta C=A A^{\mathrm{T}},
$$


where $\boldsymbol{A}$ is a lower triangular matrix and $\boldsymbol{A}^{\mathrm{T}}$ is its transposed expression. The lower triangular matrix $\boldsymbol{A}$ has 21 entries, and can be obtained using a random vector field. Let $\bar{A}$ and $\boldsymbol{A}^{\prime}$ be respectively the mean and fluctuation of the random vector field, with $\boldsymbol{A}=\overline{\boldsymbol{A}}+\boldsymbol{A}^{\prime}$. We assume that the random vector field $\boldsymbol{A}^{\prime}$ can be described as a homogeneous random field. Because of the existence of the lower bound, the expectation of the norm of the generated tensor inverse $C_{\mathrm{M}}^{-1}$ exists as demonstrated in Appendix $\mathrm{B}$, ensuring the convergence of the SFEM $[29,30]$.

The spectral representation method [36] is applied to generate the required random field $\boldsymbol{A}^{\prime}(\boldsymbol{x}, \boldsymbol{\theta})$ based on the known cross-correlation matrix $\boldsymbol{R}_{\boldsymbol{A}^{\prime}}(\boldsymbol{\tau})(27)$, see Section 3.3. Although the cross-correlation matrix $\boldsymbol{R}_{\boldsymbol{A}^{\prime}}(\boldsymbol{\tau})$ is only computed in a limited spatial distance, a large random field can be generated easily by considering a zero-padding once $\boldsymbol{R}_{\boldsymbol{A}^{\prime}}(\boldsymbol{\tau})$ reaches zero. Details on the generation process of the random field $\boldsymbol{A}^{\prime}(\boldsymbol{x}, \boldsymbol{\theta})$ are reported in Appendix C. In this paper we assume that the random vector field $\boldsymbol{A}^{\prime}$ can be described as a homogeneous Gaussian random field. This assumption will be shown to be accurate in the studied case in the next paragraph, but the developed method remains valid for another random field assumption, and we discuss in Appendix $\mathrm{C}$ how nonGaussian random fields can be considered.

Samples of the elasticity tensor can then be obtained from Eqs. (35) and (36) as

$$
\boldsymbol{C}_{\mathrm{M}}(\boldsymbol{x}, \boldsymbol{\theta})=\boldsymbol{C}_{\mathrm{L}}+\left(\overline{\boldsymbol{A}}+\boldsymbol{A}^{\prime}(\boldsymbol{x}, \boldsymbol{\theta})\right)\left(\overline{\boldsymbol{A}}+\boldsymbol{A}^{\prime}(\boldsymbol{x}, \boldsymbol{\theta})\right)^{T} .
$$

\subsection{Application to the poly-silicon case}

Table 3: Errors in the material properties mean values and standard deviations obtained with the spectral generator as compared to the values obtained directly from the SVE realizations, for an SVE length of $0.4 \mu \mathrm{m}$

\begin{tabular}{|c||c|c|}
\hline Material property & Error in the mean & $\begin{array}{c}\text { Error in the } \\
\text { standard deviation }\end{array}$ \\
\hline Young's modulus $E_{x}$ & $0.026 \%$ & $0.97 \%$ \\
\hline Poisson ratio $\nu_{y x}$ & $0.043 \%$ & $1.48 \%$ \\
\hline Shear modulus $G_{x z}$ & $0.072 \%$ & $10.09 \%$ \\
\hline
\end{tabular}

In this section we generate a random field using the SVE realizations described in Section 3.4, and the generated elasticity tensor random field is compared with the distribution directly obtained from the sample realizations.

In Fig. 8, the histograms of $E_{x}$, the Young's modulus along the SVE length direction, extracted from the elasticity tensor $\boldsymbol{C}_{\mathrm{M}}$ are compared for different SVE lengths. The distributions obtained with the generator are qualitatively in good agreements with the ones obtained from the SVE realizations. The same conclusion holds for the histograms of the Poisson ratio and of the shear modulus respectively shown in Figs. 9(a) and 9(b). The errors on the mean and the standard deviation of the material distribution resulting from the generator are reported in Tab. 3 for an SVE length of $0.4 \mu \mathrm{m}$. While good agreements 


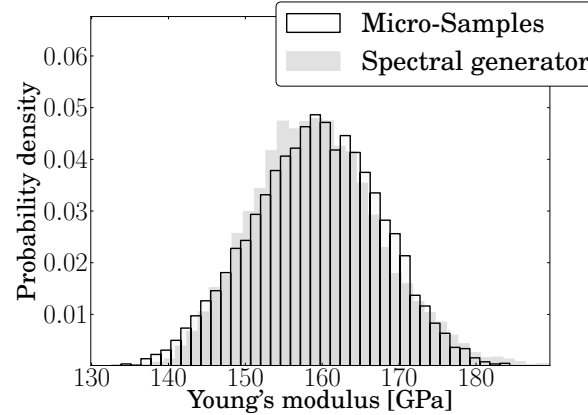

(a) $l_{\mathrm{SVE}}=0.1 \mu \mathrm{m}$

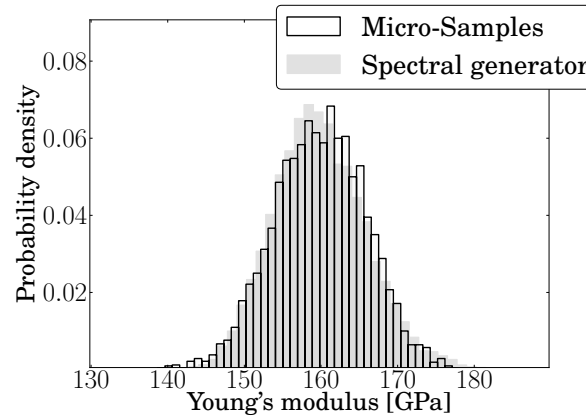

(c) $l_{\mathrm{SVE}}=0.4 \mu \mathrm{m}$

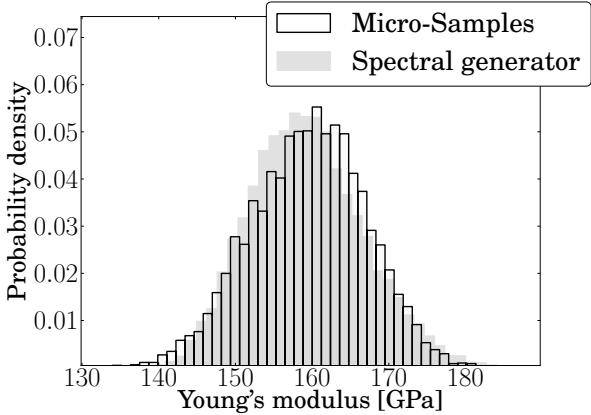

(b) $l_{\mathrm{SVE}}=0.2 \mu \mathrm{m}$

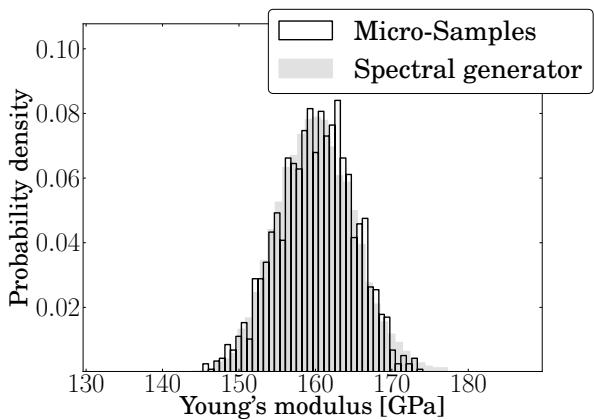

(d) $l_{\mathrm{SVE}}=0.6 \mu \mathrm{m}$

Figure 8: Comparison of the Young's modulus $E_{x}$ histograms obtained directly from the SVE realizations and with the spectral generator for different SVE lengths ( $x$ is along the SVE length)

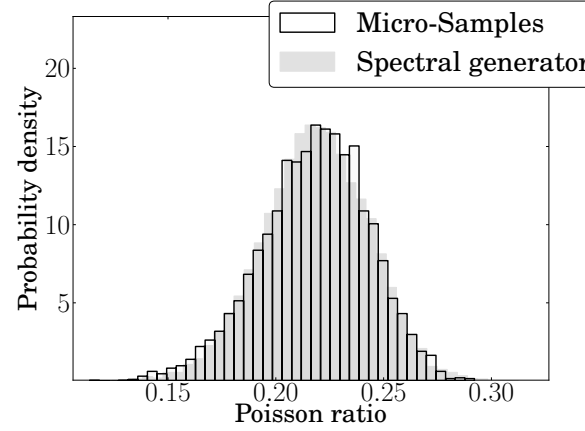

(a) Poisson ratio in the $y x$-direction

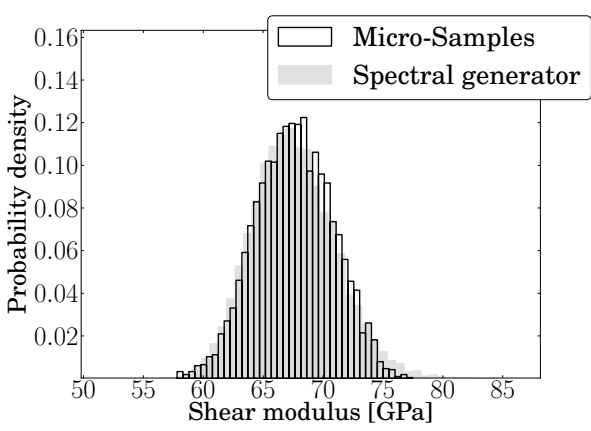

(b) Shear modulus in the $x z$-direction

Figure 9: Comparison of the material properties histograms obtained directly from the SVE realizations and with the spectral generator for an SVE length of $0.4 \mu \mathrm{m}$ : (a) $y x$-Poisson ratio, and (b) $x z$-shear modulus ( $x$ is along the SVE length, $y$ along the width, and $z$ along its height) 


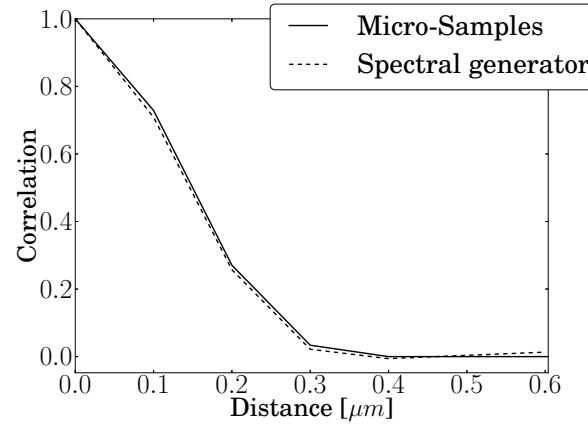

(a) Young's modulus along the $x$ direction

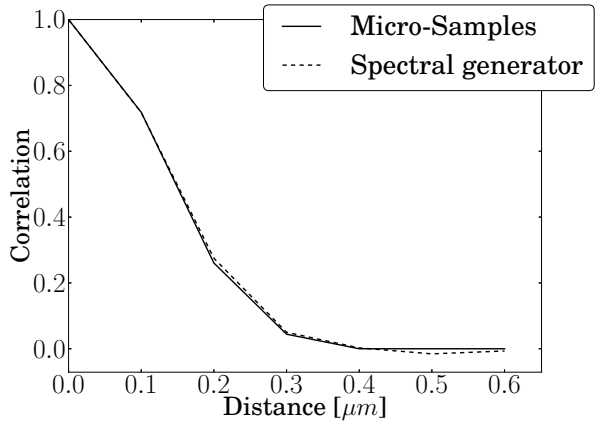

(b) Shear modulus in the $x z$-direction

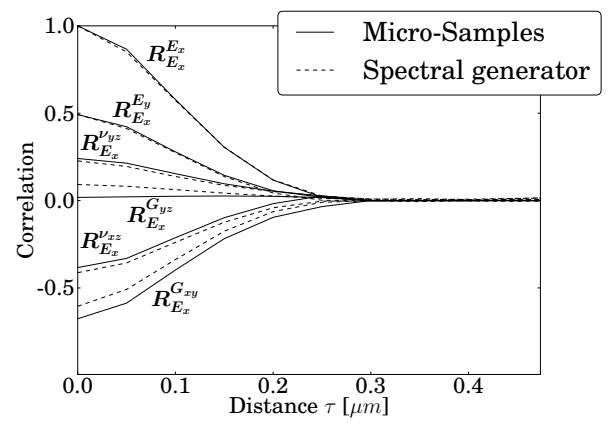

(c) Cross-correlation

Figure 10: Comparison of the 1D spatial correlations obtained directly from the SVE realizations and with the spectral generator: (a) Auto-correlation of the Young's modulus along the $x$-direction for an SVE length of $0.4 \mu \mathrm{m}$, (b) auto-correlation of the $x z$-shear modulus for an SVE length of $0.4 \mu \mathrm{m}$ ( $x$ is along the SVE length, $y$ along the width, and $z$ along its height), and (c) Cross-correlations of the the Young's modulus along the $x$-direction with other material constants, for an SVE length of $0.1 \mu \mathrm{m}$.

are obtained for the Young's modulus and the Poisson ratio, a higher difference is obtained for the shear modulus. As, in our case, the Young's modulus is the main parameter governing the structural problem, the accuracy of the random generator is satisfying for our application. The skewness of the Young's modulus distribution, $\gamma_{1_{E_{x}}}=\frac{\mathbb{E}\left[\left(E_{x}-\mathbb{E}\left[E_{x}\right]\right)^{3}\right]}{\sigma_{E_{x}}^{3}}$, obtained from the micro-samples and from the generator is -0.11 and 0.26 , respectively. Both characterize a distribution close to symmetry, although the use of a lower bound for the generator induces a positive value. The peak intensity of the Young's modulus distribution is characterized by the kurtosis, $\beta_{E_{E_{x}}}=\frac{\mathbb{E}\left[\left(E_{x}-\mathbb{E}\left[E_{x}\right]\right)^{4}\right]}{\sigma_{E_{x}}^{4}}$ and is found to be 2.93 and 3.02 , for the distribution obtained from the micro-samples and from the generator, respectively. The kurtosis is thus found to be in good agreement justifying the use of the developed generator. Nevertheless different non-Gaussian fields could be considered to improve the prediction of the shear modulus, see the discussion in the introduction and in Appendix C. 


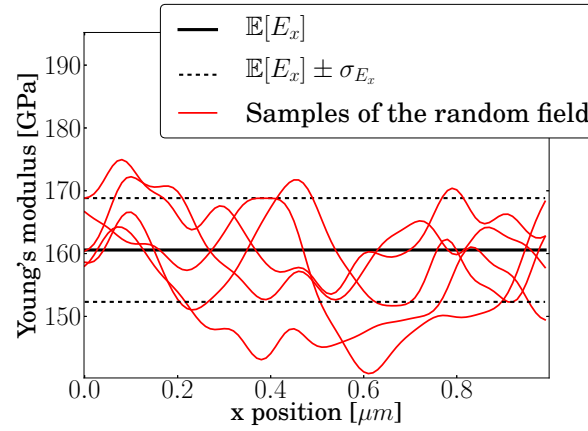

(a) $l_{\mathrm{SVE}}=0.1 \mu \mathrm{m}$

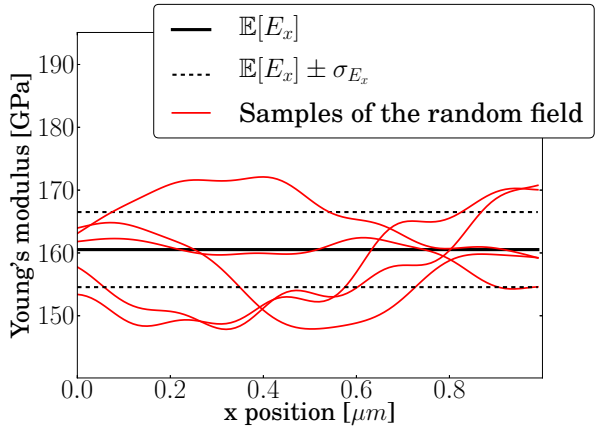

(b) $l_{\mathrm{SVE}}=0.4 \mu \mathrm{m}$

Figure 11: Five realizations of the meso-scale random field obtained with (a) an SVE length $l_{\mathrm{SVE}}=0.1 \mu \mathrm{m}$ and (b) an SVE length $l_{\mathrm{SVE}}=0.4 \mu \mathrm{m}$ : meso-scale $x$-evolution of the Young's modulus. The bold line correspond to $\mathbb{E}\left[E_{x}\right]$, the average meso-scale value of the Young's modulus, andthe dotted lines include $\sigma_{E_{x}}$, its standard deviation.

Finally, Fig. 10(a) and 10(b) respectively compare the 1D-spatial autocorrelation of the Young's modulus and of shear modulus obtained directly from the SVE computations (with the zero-padding) and with the generator for an SVE length of $0.4 \mu \mathrm{m}$. The two curves are almost identical. Figure 10(c) compares the different cross-correlations, already studied in Fig. 7(a), obtained with both the micro-samples (with the zero-padding) and with the generator for an SVE length of $0.1 \mu \mathrm{m}$. The behaviours obtained with the generator is in good agreement with the original distribution.

Once the generator has been numerically verified for the different SVE lengths $l_{\mathrm{SVE}}$, it can be used to provide several meso-scale random field realizations at a lower cost than solving the SVEs at each sampling point. As a way of illustration, Figs. 11(a) and (b) represent five realizations of the Young's modulus meso-scale random field along a distance of $1 \mu \mathrm{m}$ obtained using the generator based on SVE lengths $l_{\mathrm{SVE}}=0.1 \mu \mathrm{m}$ and $l_{\mathrm{SVE}}=0.4 \mu \mathrm{m}$, respectively. The $x$-evolution of the Young's modulus is compared to $\mathbb{E}\left[E_{x}\right]$ its average mesoscale value and to $\sigma_{E_{x}}$, its standard deviation. The effect of the SVE length when propagating the meso-scale uncertainties to the macro-scale will be studied in the next section, in which the results are shown to converge with the macro-scale finite element mesh size (for all $l_{\mathrm{SVE}}$ ).

\section{Stochastic finite element method: from the meso-scale to the struc- tural-scale}

In this section we propagate the uncertainties of the random field generated at the meso-scale in Section 4 to the structural-scale using the stochastic finite element method described in Section 2. The accuracy and efficiency of the resulting 3 -scale stochastic method is ascertained by comparing the predictions 
with the results obtained from direct MC simulations on a full discretization of the structure, i.e. for which the grains are meshed individually.

\subsection{The stochastic finite element problem}

As an illustration example we consider the problem of micro-beam resonators made of poly-silicon. In particular we study the distributions of its first three resonance frequencies ${ }^{4}$.

We first consider micro-beams of dimensions $3.2 \mu \mathrm{m} \times 0.5 \mu \mathrm{m} \times 0.1 \mu \mathrm{m}$. The structural-scale has a size of the first mode, which is four times the size of the beam: $l_{\text {macro }}=12.8 \mu \mathrm{m}$. As the largest SVE length considered is $0.6 \mu \mathrm{m}$, we satisfy the length scale separation $l_{\text {meso }}<<l_{\text {macro }}$ in this application. A short length of the beam is chosen in order to be able to obtain a reference solution of the problem by direct MC simulations of the poly-crystalline structure in a reasonable time. The Monte-Carlo simulations are then applied on a finiteelement model of about $\approx 20000$ quadratic tetrahedral finite elements.

To apply the stochastic 3 -scale method, the micro-beam is discretized using Timoshenko beam finite elements. The locking-free finite-element formulation using the interdependent interpolation model developed in [46] is used in this work.

In the context of the point discretization method, the Young's and shear moduli of an element are obtained from the generated meso-scale random elasticity tensor evaluated at the element center point. The equations of the stochastic finite element problem (5), with $\boldsymbol{f}=0$ for the free vibration problem, can then be directly obtained and solved for each realization $\boldsymbol{\theta}$. A Monte-Carlo analysis is then applied to compute the distribution of the micro-beam resonance frequencies.

In order to evaluate the existing bias from the deterministic FE models, i.e. by using 1D-beam and 3D finite elements, the beam is studied using an isotropic material (Young's modulus of $160 \mathrm{GPa}$ and shear modulus of $68 \mathrm{GPa}$ ). The number of element for both models is enough to ensure the convergence of (at least) the first three eigen-frequencies with respect to the element-size to reach an accuracy better than $0.5 \%$. With the $1 \mathrm{D}$-beam finite elements the first resonance frequency is found to be $13.068 \mathrm{MHz}$ while it is found to be 13.106 $\mathrm{MHz}$ with the 3D finite elements. The difference of $0.29 \%$ is lower than the uncertainties resulting from the polycrystalline material organization.

Finally, all the MC simulations results are presented for a sufficiently high number of generated samples, i.e. respectively at least 5000 and 3500 for the 3 -scale and the direct procedures, to ensure the convergence of the average

\footnotetext{
${ }^{4}$ The homogenization process described in Section 3 does not account for the dynamic effects. This assumption is valid as the time-scales separation of the problem exists. Indeed the characteristic time of the dynamic problem $t_{\text {macro }}$ depends on the first resonance frequency which is approximately $13 \mathrm{MHz}$ as it will be shown. Therefore $t_{\text {macro }} \approx 7 \cdot 10^{-8} \mathrm{~s}$. As the speed of sound in Silicon is about $8433 \mathrm{~ms}^{-1}$ and as the size of the largest SVE is $0.6 \mu \mathrm{m}$, the characteristic time of the micro problem is $t_{\text {micro }} \approx 7 \cdot 10^{-11} \mathrm{~s}$. As there are several orders of magnitude between the time-scales, one can neglect the stress wave problem within an SVE.
} 


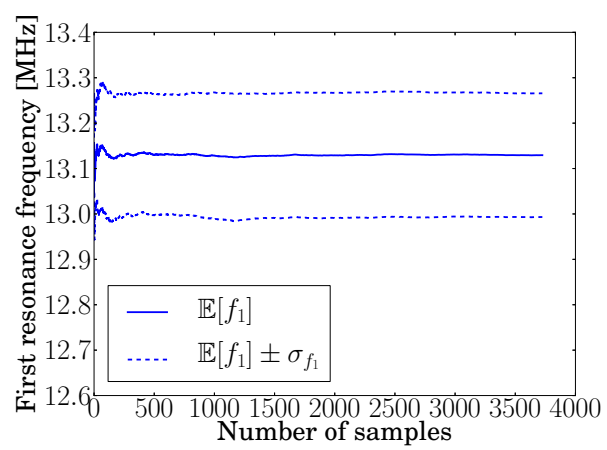

(a) Direct finite elements

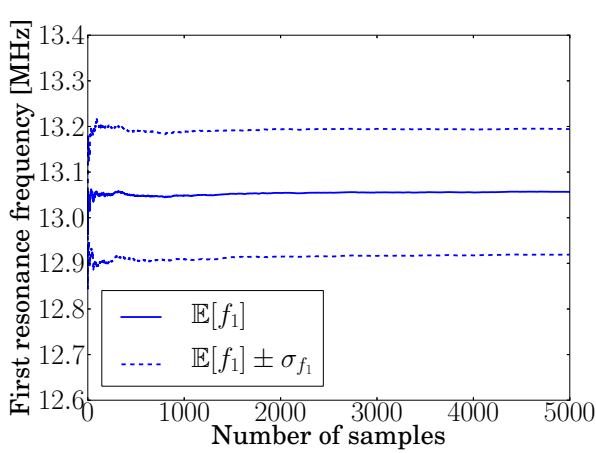

(b) 3-scale approach

Figure 12: Convergence study of the MC simulations of the $3.2 \mu \mathrm{m}$-long beam for both (a) the direct finite element approach and (b) the 3 -scale approach $\left(l_{\mathrm{SVE}}=0.4 \mu \mathrm{m}\right.$ and a macro-mesh size of $0.25 \mu \mathrm{m}$ )

resonance frequencies and of their standard deviations. As an example, in the particular case which will be study hereafter $\left(3.2 \mu \mathrm{m}\right.$-long beam; $l_{\mathrm{SVE}}=0.4 \mu \mathrm{m}$ and a macro mesh size of $0.25 \mu \mathrm{m}$ for the 3 -scale simulations), Figs. 12(a) and (b) report, for respectively the direct finite element simulations and the 3 -scale approach, the convergence of these values with respect to the number of samples. It can be seen that, beyond 1500 samples, the MC simulations have converged.

\subsection{Effects of the SVE and structural mesh sizes}

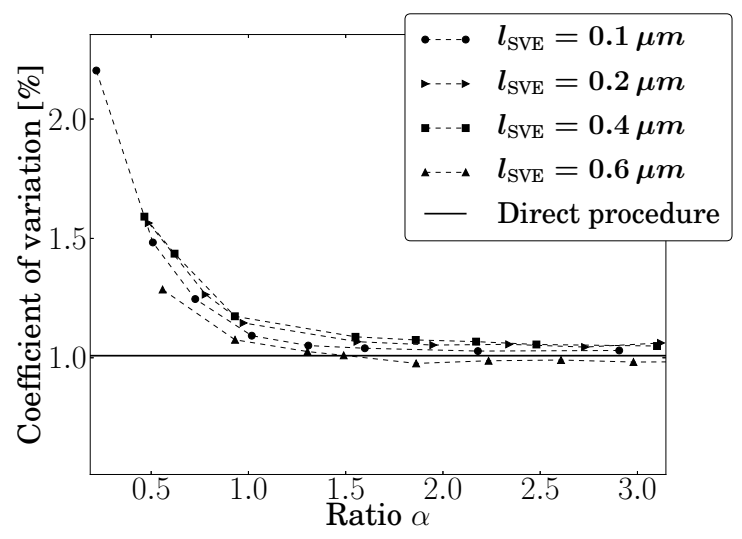

Figure 13: The COV of the first resonance frequency for a $3.2 \mu \mathrm{m}$-long beam for different SVE lengths and finite element mesh sizes in terms of $\alpha=\frac{l_{E_{x}}}{l_{\text {mesh }}}$, the ratio between the correlation length of the Young's modulus, $l_{E_{x}}$, and the mesh size, $l_{\text {mesh }}$

The developed 3-scale stochastic method is applied for different finite element 
discretizations (mesh sizes $\left.l_{\text {mesh }}\right)$ and SVE lengths $\left(l_{\mathrm{SVE}}\right)$.

In order to illustrate the effect of the mesh size, the evolution of the predicted first resonance frequency COV is reported in Fig. 13 in terms of the ratio $\alpha=\frac{l_{E_{x}}}{l_{\operatorname{mesh}}}$ between the correlation length of the Young's modulus, $l_{E_{x}}$, see Tab. 1 , and the mesh size, $l_{\text {mesh }}$. In the cases of $\alpha<1$, the mesh size is larger than the correlation length, and the stochastic finite element method suffers from a lack of accuracy $[4,5]$. Indeed, the predicted COV is found to depend on the mesh-size -and thus on SVE length through the correlation length $l_{E_{x}}-$ and to be higher than the reference solution. This is physically explained by the fact that, in this case, the finite element size is larger than its associated SVE and the amount of grains associated to each finite element is underestimated, leading to an over-prediction of the uncertainties. When refining the finite element mesh, i.e. for $\alpha$ larger than one, the 3 -scale predictions are found to converge toward the reference solution as expected with the use of the SFEM. In these cases, the SVEs are of size comparable to or larger than the finite-element sizes and the existing spatial correlation between the SVEs defined on the finite elements accounts for the change of meso-scale distribution arising with a change of SVE length. As we are using the center point method to discretize the random field, the 3 -scale method converges by overestimating the uncertainties, in agreement with the literature [38].

Table 4: Comparison of the first three mean resonance frequencies obtained with the 3-scale stochastic method and with the direct MC simulations

\begin{tabular}{|c||c|c|c|}
\hline Mode & 3-scale approach & Direct procedure & Relative difference \\
\hline $\mathbf{1}$ & $13.055 \mathrm{MHz}$ & $13.129 \mathrm{MHz}$ & $0.57 \%$ \\
\hline $\mathbf{2}$ & $64.448 \mathrm{MHz}$ & $64.115 \mathrm{MHz}$ & $0.51 \%$ \\
\hline $\mathbf{3}$ & $81.554 \mathrm{MHz}$ & $81.896 \mathrm{MHz}$ & $0.42 \%$ \\
\hline
\end{tabular}

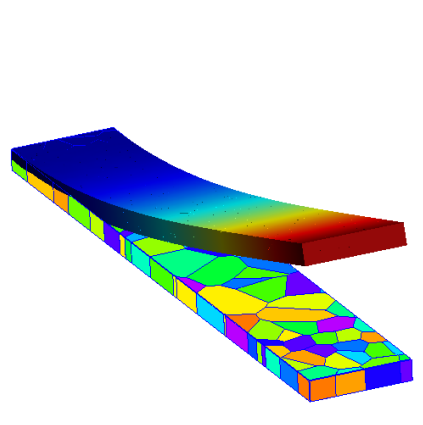

(a) Direct finite element sample

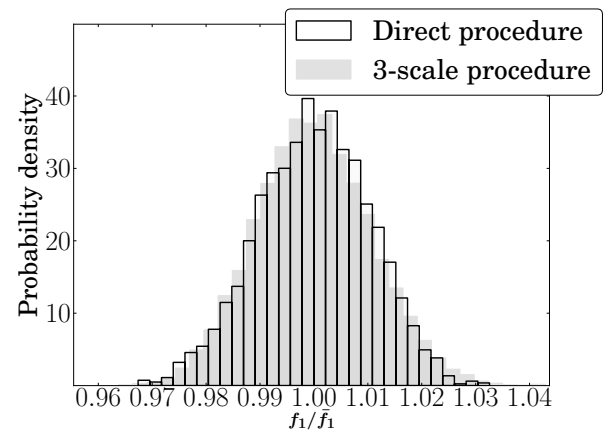

(b) Resonance frequency histograms

Figure 14: Comparison of the first resonance frequency histograms obtained with the 3-scale stochastic method and with the direct MC simulations. (a) Example of a direct finite element simulation. (b) Resonance frequency histograms 


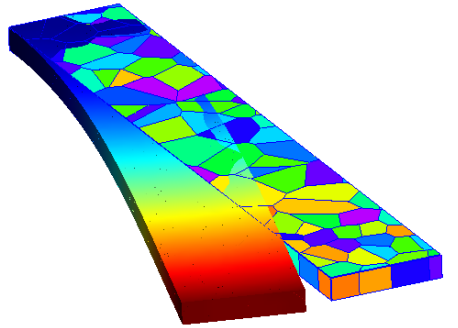

(a) Direct finite element sample

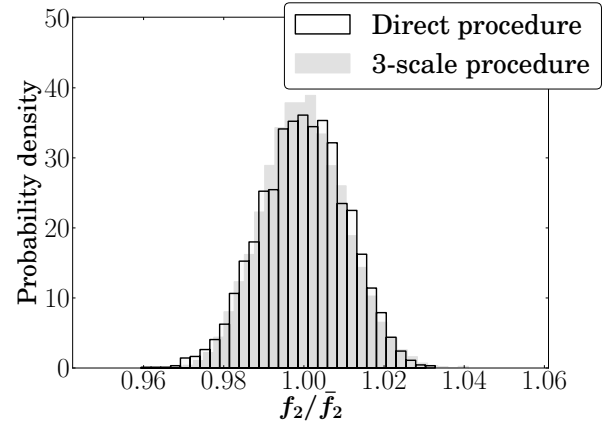

(b) Resonance frequency histograms

Figure 15: Comparison of the second resonance frequency histograms obtained with the 3scale stochastic method and with the direct MC simulations. (a) Example of a direct finite element simulation. (b) Resonance frequency histograms.

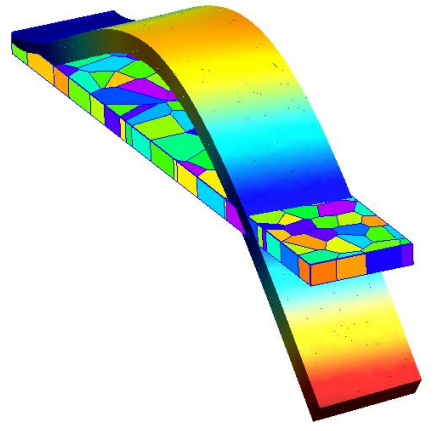

(a) Direct finite element sample

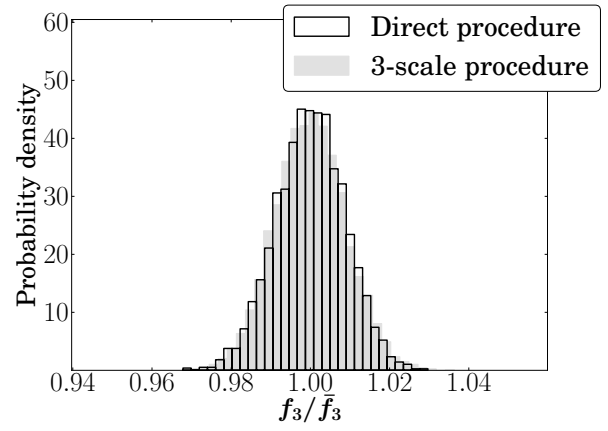

(b) Resonance frequency histograms

Figure 16: Comparison of the third resonance frequency histograms obtained with the 3-scale stochastic method and with the direct MC simulations. (a) Example of a direct finite element simulation. (b) Resonance frequency histograms

The first three resonance frequencies histograms obtained with the 3-scale stochastic method and with the direct MC simulations are reported in Figs. 14 - 16 along with the illustration of the resonance modes. For the 3 -scale model, the considered SVE length and mesh size are respectively $0.4 \mu \mathrm{m}$ and $0.27 \mu \mathrm{m}$. The resonance frequencies obtained with both procedures are normalized with their corresponding mean reported in Tab. 4. The histograms obtained by the stochastic 3 -scale method are in good agreement with the reference results obtained by the direct MC simulations. The difference in the mean frequency mainly results from the bias between the models based on 3D finite elements and the 1D-beam finite elements. For the second resonance frequency, the resonancemode is out of plane and as the grains are not columnar, the Young's modulus is not uniform along the vibrating direction either. A second-order homogenization 
[44] should thus be used for that bending mode, however the predictions are still in good agreement.

When comparing the computational times, the resolution of the full model represents a computation cost around nine hours per sample on a $3.4 \mathrm{GHz}$ $\mathrm{CPU}$ to be compared to a few milliseconds per sample for the 3 -scale procedure with the mesh size equal to $0.2 \mu \mathrm{m}$. However in order to define the generator, SVE homogenization had to be computed, which is also time consuming. The extraction of the homogenized material tensor of a sample for a $0.2 \mu \mathrm{m}$-long SVE requires about 97 seconds. Approximately 50 hours are thus required to define the meso-scale random field. This remains much shorter than the time required to compute samples with the direct procedure. Moreover, the computed SVE information can be used for different meso-to-macro scale problems. If the size of the structural problem were to be increased, the interest of the proposed stochastic 3-scale process would become higher as direct MC simulations would become unreachable. Finally, using a smaller SVE reduces the homogenization computational time, but requires a finer mesh size at the structural scale, or an increase in the number of integration points.

\subsection{Effect of the micro-beam length}

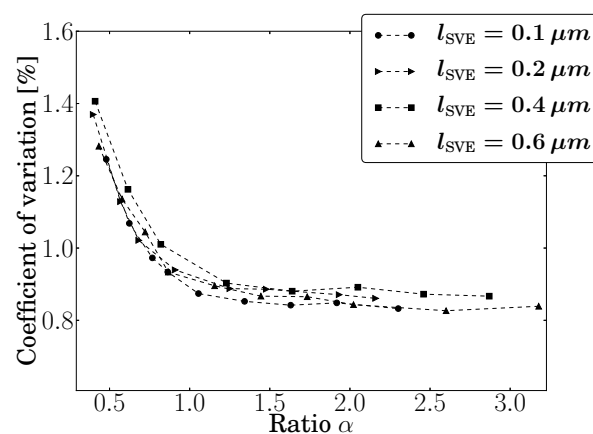

(a) $4.8 \mu \mathrm{m}$-long beam

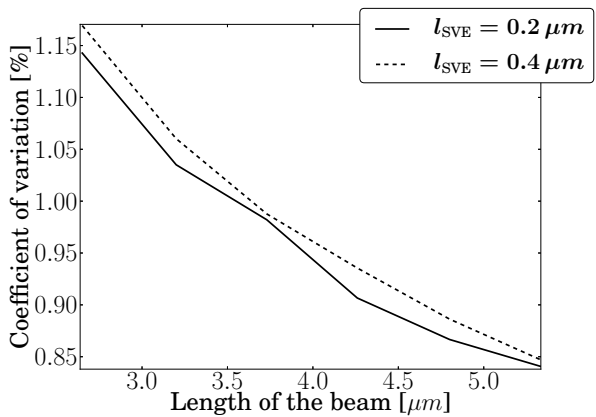

(b) Influence of the beam length

Figure 17: Results for different beam lengths. (a) The COV of the first resonance frequency for a $4.8 \mu \mathrm{m}$-long beam for different SVE lengths and finite element mesh sizes in terms of $\alpha=\frac{l_{E_{x}}}{l_{\text {mesh }}}$, the ratio between the correlation length of the Young's modulus, $l_{E_{x}}$, and the mesh size, $l_{\text {mesh }}$. (b) Evolution of the first resonance frequency COV with respect to the beam length for different SVE lengths.

Results were also computed for different lengths of the micro-beam. Figure 17(a) illustrates the evolution with $\alpha$ of the coefficient of variation obtained with different SVE lengths for a $4.8 \mu \mathrm{m}$-long micro-beam. It can be seen that the uncertainty is smaller than for the $3.2 \mu \mathrm{m}$ beam: $C O V \approx 0.9 \%$ vs. $C O V \approx 1 \%$. The influence of the beam length is depicted in Fig. 17(b) for two SVE lengths: 0.2 and $0.4 \mu \mathrm{m}$. The finite element mesh is such that a constant ratio $\alpha$ is obtained (respectively, $\alpha=2.3$ and $\alpha=1.9$ ). As expected, the coefficient of 
variation of the first resonance frequency decreases with an increase of the length of the beam.

\section{Conclusions and perspectives}

A stochastic 3-scale approach to propagate uncertainties from the microstructure to a macro-scale quantity of interest was developed in this paper. Polycrystalline materials were considered and two sources of uncertainties were involved: the grain geometries and orientations.

In this proposed stochastic 3-scale approach, at the micro-level a structuralpoint is viewed as the center of a stochastic volume element. The SVEs were generated under the form of a Voronoï tessellation with a random orientation for each silicon grain. As SVEs are not statistically representative, a Monte-Carlo procedure combined with a homogenization technique allowed a distribution of the apparent material tensor of the meso-scale SVE to be estimated. The correlation between the meso-scale material tensors of two SVEs at a given distance could also be evaluated. An elasticity tensor generator at the meso-scale was built based on the knowledge of the random field structure obtained from the SVE simulations, using a spectral representation method. The generator accounts for a lower bound of the meso-scale material tensor in order to ensure the existence of the expectation of the generated material tensor inverse. Using the random meso-scale field obtained with the meso-scale generator, a SFEM was then applied at the structural-scale to predict the probabilistic behavior of the MEMS resonator.

Probabilistic predictions obtained with the 3 -scale stochastic method were then compared to direct $\mathrm{MC}$ simulations of the micro-beam first three resonance frequencies, in which the whole micro-structure is meshed. The two methods gave similar results, the 3 -scale procedure being much less expensive in terms of computational effort. Thus the proposed stochastic 3-scale method offers an alternative to direct $\mathrm{MC}$ simulations for large problems for which the direct procedure is not feasible.

In the future, it is intended to enrich the model description by considering preferred grain orientations, imperfection on the beam thickness ... To account for more complex problems and geometries, the meso-scale to macro-scale transition will be applied in a 3D setting (the generated random field is already yielding 3D elasticity tensors). Moreover the case for which the structural-scale finite-elements are larger than the correlation length will be investigated by using other random field discretization methods in the 3D bulk elements.

The phenomenon of thermo-elastic damping [39], which has an important effect on the dynamic behavior of micro-resonators, will also be studied through the proposed three-scale process. This will be achieved by first extending the developed stochastic multi-scale method to thermo-elasticity and then by considering thermo-elastic damping in the 3D stochastic finite element analyses. The validation of the proposed process against experimental data will then become feasible by measuring the vibrating properties of poly-silicon MEMS resonators using a laser-vibrometer. 
In another context, it is also intended to formulate the 3-scale method recently proposed in [47] for the fracture of poly-silicon micro films in a stochastic way.

\section{Appendix A. Poisson-Voronoï tessellation and SVEs extraction}

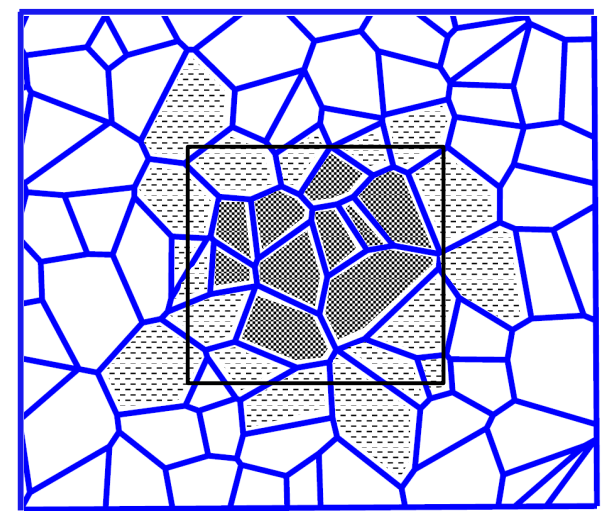

Figure A.18: Extraction of an SVE from a Poisson-Voronoï tessellation: the checked grains correspond to polygons not intersecting the SVE boundary and the dashed grains correspond to polygons intersecting the SVE boundary.

In this section we explain how to build the Poisson-Voronoï tessellation and how to construct the SVEs required for the moving window technique, see Section 3.3. A 2D Poisson-Voronoï tessellation can be generated using the "scipy.spatial" python library called "Voronoi" from random seeds which follow a uniform distribution. The resulting large Voronoï tessellation is described by the information of vertexes and lines of each polygon. One rectangular parallelepiped SVE can then be extracted from the large 2D Voronoï tessellation, see Fig. A.18, by going through the following steps:

- Looping on all the polygons of the large Voronoï tessellation, the polygons can be categorized into three groups: the polygons with all their vertexes inside the SVE - checked polygons in Fig. A.18, the polygons with a part of their vertexes inside the SVE -dashed polygons in Fig. A.18, and the polygons with none of their vertexes inside the SVE;

- For the first group of polygons, all the information (vertexes and lines) is kept for the SVE construction, while for the third group of polygons, all the information is discarded;

- For the polygons of the second group, their vertexes outside of the SVE are replaced by the intersection points of their lines with the boundaries of the SVE, their intersected lines are shortened, the lines outside of the SVE are discarded, and their lines are completed by the boundary parts of the SVE; 
- A new set of polygons, with their associated vertexes and lines, is then defining the SVE; a 2D mesh conforming with these new polygons can then be generated using GMSH [48], and an extrusion follows to obtain a 3D columnar SVE meshed with quadratic tetraedra, see Fig. 3;

- Fnally each extruded polygon is associated to a domain having its own anisotropic material law defined by its orientation angles, allowing the definition of grains with different orientations within the SVE.

\section{Appendix B. Notes on the random field generator}

As described in Section 4, elasticity tensors are modelled as a random field obtained from a generator. A lower bound is introduced so that positive semidefinite tensors $\Delta \boldsymbol{C}=\boldsymbol{C}_{\mathrm{M}}-\boldsymbol{C}_{\mathrm{L}}$ are generated. As one can read in [30], the existence of the expectation of the norm of the generated elasticity tensor inverse is a fundamental property of the matrix random field. This existence can be proven thanks to the lower bound of the elasticity tensor.

Indeed, this fundamental property is written as:

$$
\mathbb{E}\left[\left(\sup _{\boldsymbol{x} \in \Omega}\left\|\left(\boldsymbol{C}_{\mathrm{M}}(\boldsymbol{x}, \boldsymbol{\theta})\right)^{-1}\right\|\right)^{2}\right]=c^{2}<+\infty,
$$

where "sup" is the supremum and $c$ is a finite positive constant. The norm operator used in Eq. (B.1) is defined as (for a square matrix) $\|\boldsymbol{A}\|=\left|\lambda_{\max }^{A}\right|$, where $\left|\lambda_{\max }^{A}\right|$ is the largest modulus of the eigen-values of $\boldsymbol{A}$ [49].

Using the compliance matrices, as $\boldsymbol{S}_{\mathrm{L}}-\boldsymbol{S}_{\mathrm{M}}(\boldsymbol{x}, \boldsymbol{\theta}) \geq 0$, from the definition of a positive semi-definite matrix, one has:

$$
\boldsymbol{z}^{T} \boldsymbol{S}_{\mathrm{L}} \boldsymbol{z}-\boldsymbol{z}^{T} \boldsymbol{S}_{\mathrm{M}}(\boldsymbol{x}, \boldsymbol{\theta}) \boldsymbol{z} \geq 0 \quad \forall \boldsymbol{z} \in \mathbb{R}^{6} .
$$

As this equation holds for every $\boldsymbol{z}$, it is also satisfied for the (normalized) eigenvector $\boldsymbol{z}_{\max }^{S_{\mathrm{M}}}(\boldsymbol{x})$ of $\boldsymbol{S}_{\mathrm{M}}(\boldsymbol{x}, \boldsymbol{\theta})$, which corresponds to the maximum eigen-value $\lambda_{\max }^{S_{\mathrm{M}}}$, yielding

$$
\left(\boldsymbol{z}_{\max }^{S_{\mathrm{M}}}(\boldsymbol{x})\right)^{T} \boldsymbol{S}_{\mathrm{L}} \boldsymbol{z}_{\max }^{S_{\mathrm{M}}}(\boldsymbol{x})-\left(\boldsymbol{z}_{\max }^{S_{\mathrm{M}}}(\boldsymbol{x})\right)^{T} \boldsymbol{S}_{\mathrm{M}}(\boldsymbol{x}, \boldsymbol{\theta}) \boldsymbol{z}_{\max }^{S_{\mathrm{M}}}(\boldsymbol{x}) \geq 0 .
$$

Using the definition of the maximum eigen-value of a positive definite real symmetric matrix, one also has

$$
\boldsymbol{z}^{T} \boldsymbol{S}_{\mathrm{L}} \boldsymbol{z} \leq \lambda_{\max }^{S_{\mathrm{L}}}\|\boldsymbol{z}\|_{2}^{2} \quad \forall \boldsymbol{z} \in \mathbb{R}^{6},
$$

Including (B.4) in (B.3) results in, as $\boldsymbol{z}_{\max }^{S_{\mathrm{M}}}$ is normalized,

$$
\lambda_{\max }^{S_{\mathrm{L}}} \geq \lambda_{\max }^{S_{\mathrm{M}}},
$$

which demonstrates the property (B.1). 


\section{Appendix C. Generation of the random field $\boldsymbol{A}^{\prime}(x, \boldsymbol{\theta})$}

In this section we detail the spectral representation method [36], which is applied to generate the required random field $\boldsymbol{A}^{\prime}(\boldsymbol{x}, \boldsymbol{\theta})$, see Section 3.3. We assume in this paper that the random field $\boldsymbol{A}^{\prime}(\boldsymbol{x}, \boldsymbol{\theta})$ is homogeneous. Its (normalized) cross-correlation matrix $\boldsymbol{R}_{\boldsymbol{A}^{\prime}}(\boldsymbol{\tau})$ (27) has been obtained through equations (35) and (36) and using the samples of the SVE elasticity tensors. Since the matrix $\boldsymbol{A}^{\prime}$ has 21 independent entries, we rewrite the random matrix $\boldsymbol{A}^{\prime}(\boldsymbol{x})$ as a vector $\left[A^{\prime(1)}(\boldsymbol{x}), A^{\prime(2)}(\boldsymbol{x}), \ldots, A^{\prime(21)}(\boldsymbol{x})\right]$. Its cross-correlation matrix $\boldsymbol{R}_{\boldsymbol{A}^{\prime}}(\boldsymbol{\tau})$ has thus a size of $21 \times 21$ entries $R_{\boldsymbol{A}^{\prime}}^{(r s)}(\boldsymbol{\tau}), r, s=1,2, \ldots, 21$.

In order to use the Discrete Fourier Transform (DFT), we first define the cross-covariance $\tilde{\boldsymbol{R}}\left[\tau_{\boldsymbol{n}}\right]$ as

$$
\tilde{R}^{(r s)}\left[\boldsymbol{\tau}_{\boldsymbol{n}}\right]=\sigma_{\boldsymbol{A}^{\prime}}^{(r)} \sigma_{\boldsymbol{A}^{\prime}}^{(s)} R_{\boldsymbol{A}^{\prime}}^{(r s)}\left(\boldsymbol{\tau}_{\boldsymbol{n}}\right),
$$

where $\sigma_{\boldsymbol{A}^{\prime}}^{(r)}$ and $\sigma_{\boldsymbol{A}^{\prime}}^{(s)}$ are the standard deviations of the $r^{\text {th }}$ and $s^{\text {th }}$ entries of $\boldsymbol{A}^{\prime}$, respectively. Considering the general $3 \mathrm{D}$ case, the set of discrete positions $\boldsymbol{\tau}_{\boldsymbol{n}}$ is defined by its vector components $\tau_{n}^{\left(n_{x} n_{y} n_{z}\right)}=\left[n_{x} \Delta \tau_{x}, n_{y} \Delta \tau_{y}, n_{z} \Delta \tau_{z}\right]$, where $\Delta \tau_{i}$, for $i=x, y, z$, is the spatial increment in each dimension $i$ and where $n_{i}=0,1,2, \ldots, N_{i}-1$, for $i=x, y, z$, with $N_{i}$ the total number of discrete points in each dimension $i$. Depending on the spatial size, $\left[l_{x}, l_{y}, l_{z}\right]$, of the required random field, we need to make sure that $N_{i} \Delta \tau_{i} \geq l_{i}$ (no sum on $i$ ). As mentioned in Section 4.2, this is achieved herein by considering a zero-padding once $\boldsymbol{R}_{\boldsymbol{A}^{\prime}}(\boldsymbol{\tau})$ reaches zero, allowing to increase the number of points $N_{i}$ as much as required. We now need to periodize $\tilde{\boldsymbol{R}}\left[\boldsymbol{\tau}_{\boldsymbol{n}}\right]$ by adding extra discrete points at its end in order to satisfy

$$
\begin{aligned}
& \tilde{\boldsymbol{R}}\left[\left(\left(N_{x}-1\right)+j\right) \Delta \tau_{x},\left(\left(N_{y}-1\right)+k\right) \Delta \tau_{y},\left(\left(N_{z}-1\right)+p\right) \Delta \tau_{z}\right]= \\
& \quad \tilde{\boldsymbol{R}}\left[\left(N_{x}-j\right) \Delta \tau_{x},\left(N_{y}-k\right) \Delta \tau_{y},\left(N_{z}-p\right) \Delta \tau_{z}\right] \\
& \quad \text { for } j=1,2, \ldots, N_{x}-1 ; k=1,2, \ldots, N_{y}-1 ; p=1,2, \ldots, N_{z}-1 .
\end{aligned}
$$

Then we have $2 N_{i}-1$ discrete points in each dimension.

The spectral density matrix $\boldsymbol{S}[\boldsymbol{m}]$ of the cross-covariance $\tilde{\boldsymbol{R}}\left[\boldsymbol{\tau}_{\boldsymbol{n}}\right]$ can now be computed using the DFT method as

$$
\begin{aligned}
& S^{(r s)}\left[\boldsymbol{m}^{\left(m_{x} m_{y} m_{z}\right)}\right]= \\
& \sum_{n_{x}=0}^{2 N_{x}-2} \sum_{n_{y}=0}^{2 N_{y}-2} \sum_{n_{z}=0}^{2 N_{z}-2} \tilde{R}^{(r s)}\left[\boldsymbol{\tau}_{\boldsymbol{n}}^{\left(n_{x} n_{y} n_{z}\right)}\right] e^{-2 \pi i\left(\frac{m_{x} n_{x}}{2 N_{x}-1}+\frac{m_{y} n_{y}}{2 N_{y}-1}+\frac{m_{z} n_{z}}{2 N_{z}-1}\right)},
\end{aligned}
$$

where the matrix $\boldsymbol{m}$ is defined by the vector components $\boldsymbol{m}^{m_{x} m_{y} m_{z}}=\left[m_{x}, m_{y}, m_{z}\right]$ with $m_{i}=0,1, \ldots, 2 N_{i}-2$ for $i=x, y, z$, and where $S\left[\boldsymbol{m}^{m_{x} m_{y} m_{z}}\right]$ is an Hermitian matrix, which can be expressed as

$$
\boldsymbol{S}\left[\boldsymbol{m}^{\left(m_{x} m_{y} m_{z}\right)}\right]=\boldsymbol{H}\left[\boldsymbol{m}^{\left(m_{x} m_{y} m_{z}\right)}\right] \boldsymbol{H}^{*}\left[\boldsymbol{m}^{\left(m_{x} m_{y} m_{z}\right)}\right],
$$


with $\boldsymbol{H}^{*}\left[\boldsymbol{m}^{\left(m_{x} m_{y} m_{z}\right)}\right]$ the conjugate transpose of the matrix $\left.\boldsymbol{H}\left[\boldsymbol{m}^{\left(m_{x} m_{y} m_{z}\right.}\right)\right]$.

Before generating the random field $\boldsymbol{A}^{\prime}$ by the spectral representation method, we define the increments of frequency, in each direction, as

$$
\Delta \kappa_{i}=\frac{1}{\left(2 N_{i}-2\right) \Delta \tau_{i}}, \quad i=x, y, z,
$$

and the matrix $\boldsymbol{\kappa}_{\boldsymbol{m}}$ of vector components $\boldsymbol{\kappa}_{\boldsymbol{m}}^{\left(m_{x} m_{y} m_{z}\right)}=\left[\kappa_{x}^{\left(m_{x}\right)}, \kappa_{y}^{\left(m_{y}\right)}, \kappa_{z}^{\left(m_{z}\right)}\right]$ is defined from (no sum on $i=x, y, z$ )

$$
\kappa_{i}^{\left(m_{i}\right)}=\left\{\begin{array}{lll}
m_{i} \Delta \kappa_{i} & \text { if } & m_{i}<\left(2 N_{i}-1\right) / 2 \\
{\left[m_{i}-\left(2 N_{i}-1\right)\right] \Delta \kappa_{i}} & \text { if } & m_{i}>\left(2 N_{i}-1\right) / 2
\end{array}\right.
$$

to avoid the failure of power and logarithm identities. Finally, the 21 components of the random field $\boldsymbol{A}^{\prime}$ are generated using

$$
\begin{array}{r}
A^{(r)}(\boldsymbol{x}, \boldsymbol{\theta})=\sqrt{2 \boldsymbol{\Delta}} \Re\left\{\sum_{s=1}^{21} \sum_{m_{x}=0}^{2 N_{x}-2} \sum_{m_{y}=0}^{2 N_{y}-2} \sum_{m_{z}=0}^{2 N_{z}-2} H^{(r s)}\left[\boldsymbol{m}^{\left(m_{x} m_{y} m_{z}\right)}\right]\right. \\
\left.\eta^{\left(s, m_{x}, m_{y}, m_{z}\right)} e^{2 \pi i\left(\boldsymbol{x} \cdot \boldsymbol{\kappa}_{m}^{\left(m_{x} m_{y} m_{z}\right)}+\theta^{\left(s, m_{x}, m_{y}, m_{z}\right)}\right.}\right\}
\end{array}
$$

where $\boldsymbol{\Delta}=\Delta \kappa_{x} \Delta \kappa_{y} \Delta \kappa_{z}, \theta^{\left(s, m_{x}, m_{y}, m_{z}\right)}$ is an independent random variable (for each $\left.s, m_{x}, m_{y}, m_{z}\right)$ uniformly distributed on $[0,1]$, and where $\eta^{\left(s, m_{x}, m_{y}, m_{z}\right)}$ can be defined in the two following ways

$$
\eta^{\left(s, m_{x}, m_{y}, m_{z}\right)}= \begin{cases}1 & \text { yields a Gaussian field only when } \\ & N_{x}, N_{y}, N_{z} \rightarrow \infty ; \\ \sqrt{-\log \varphi^{\left(s, m_{x}, m_{y}, m_{z}\right)}} & \text { yields a Gaussian field when } \\ & \varphi^{\left(s, m_{x}, m_{y}, m_{z}\right)} \text { is uniformly } \\ & \text { distributed on }[0,1] .\end{cases}
$$

In this work, the first case is considered.

In order to generate a non-Gaussian random field, the described method is first apply to generate an intermediate Gaussian random field. Afterward, this Gaussian stochastic vector field can be transformed into a non-Gaussian random field using proper mapping techniques, see for example [50].

\section{Acknowledgment}

The research has been funded by the Walloon Region under the agreement no 1117477 (CT-INT 2011-11-14) in the context of the ERA-NET MNT framework.

\section{References}

[1] R. Ghanem, P. Spanos, Stochastic Finite Elements: A Spectral Approach, Springer Verlag, 1991. 
[2] O. Lemaitre, O. Knio, Spectral methods for uncertainty quantification with applications to computational fluid dynamics, Springer, 2010.

[3] G. Stefanou, The stochastic finite element method: Past, present and future, Computer Methods in Applied Mechanics and Engineering 198 (912) (2009) 1031 - 1051, ISSN 0045-7825, doi: http://dx.doi.org/10.1016/j.cma.2008.11.007.

[4] M. Shinozuka, G. Deodatis, Response Variability Of Stochastic Finite Element Systems, Journal of Engineering Mechanics 114 (3) (1988) 499-519, doi:10.1061/(ASCE)0733-9399(1988)114:3(499).

[5] T. Harada, M. Shinozuka, The Scale of Correlation for Stochastic FieldsTechnical Report, Department of Civil Engineering and Engineering Mechanics, Columbia University, New York, NY .

[6] S. Baxter, L. Graham, Characterization of Random Composites Using Moving-Window Technique, Journal of Engineering Mechanics 126 (4) (2000) 389-397, doi:10.1061/(ASCE)0733-9399(2000)126:4(389).

[7] H. G. Matthies, C. E. Brenner, C. G. Bucher, C. Guedes Soares, Uncertainties in probabilistic numerical analysis of structures and solids-Stochastic finite elements, Structural Safety 19 (3) (1997) 283 - 336, ISSN 0167-4730, doi:http://dx.doi.org/10.1016/S0167-4730(97)00013-1, devoted to the work of the Joint Committee on Structural Safety.

[8] F. Yamazaki, A. Member, M. Shinozuka, G. Dasgupta, Neumann Expansion for Stochastic Finite Element Analysis, Journal of Engineering Mechanics 114 (8) (1988) 1335-1354, doi:10.1061/(ASCE)07339399(1988)114:8(1335).

[9] M. Shinokuza, C. J. Astill, Random Eigenvalue Problems in Structural Analysis, AIAA Journal 10 (4) (1972) 456-462, ISSN 0001-1452, doi: $10.2514 / 3.50119$.

[10] P. Castañeda, P. Suquet, Nonlinear Composites, in: E. van der Giessen, T. Y. Wu (Eds.), Advances in Applied Mechanics, vol. 34 of Advances in Applied Mechanics, Elsevier, 171 - 302, doi:10.1016/S0065-2156(08)70321$1,1997$.

[11] I. Doghri, A. Ouaar, Homogenization of two-phase elasto-plastic composite materials and structures: Study of tangent operators, cyclic plasticity and numerical algorithms, International Journal of Solids and Structures 40 (7) (2003) 1681 - 1712, ISSN 0020-7683, doi:10.1016/S0020-7683(03)00013-1.

[12] L. Wu, L. Noels, L. Adam, I. Doghri, A combined incremental-secant meanfield homogenization scheme with per-phase residual strains for elastoplastic composites, International Journal of Plasticity 51 (2013) 80 - 102, ISSN 0749-6419, doi:10.1016/j.jjplas.2013.06.006. 
[13] H. Moulinec, P. Suquet, A FFT-Based Numerical Method for Computing the Mechanical Properties of Composites from Images of their Microstructures, in: R. Pyrz (Ed.), IUTAM Symposium on Microstructure-Property Interactions in Composite Materials, vol. 37 of Solid Mechanics and Its Applications, Springer Netherlands, ISBN 978-94-010-4031-0, 235-246, doi: 10.1007/978-94-011-0059-5_20, 1995.

[14] S. Ghosh, K. Lee, S. Moorthy, Multiple scale analysis of heterogeneous elastic structures using homogenization theory and voronoi cell finite element method, International Journal of Solids and Structures 32 (1) (1995) 27 62, ISSN 0020-7683, doi:http://dx.doi.org/10.1016/0020-7683(94)00097-G.

[15] J. Michel, H. Moulinec, P. Suquet, Effective properties of composite materials with periodic microstructure: a computational approach, Computer Methods in Applied Mechanics and Engineering 172 (1-4) (1999) 109 - 143, ISSN 0045-7825, doi:10.1016/S0045-7825(98)00227-8.

[16] C. Miehe, A. Koch, Computational micro-to-macro transitions of discretized microstructures undergoing small strains, Archive of Applied Mechanics 72 (4-5) (2002) 300-317, ISSN 0939-1533, doi:10.1007/s00419-0020212-2.

[17] V. Kouznetsova, W. A. M. Brekelmans, F. P. T. Baaijens, An approach to micro-macro modeling of heterogeneous materials, Computational Mechanics 27 (1) (2001) 37-48, ISSN 0178-7675, doi:10.1007/s004660000212.

[18] T. Kanit, S. Forest, I. Galliet, V. Mounoury, D. Jeulin, Determination of the size of the representative volume element for random composites: statistical and numerical approach, International Journal of Solids and Structures 40 (13-14) (2003) 3647-3679, ISSN 0020-7683, doi:10.1016/S00207683(03)00143-4.

[19] M. Geers, V. Kouznetsova, W. Brekelmans, Multi-scale computational homogenization: Trends and challenges, Journal of Computational and Applied Mathematics 234 (7) (2010) 2175 - 2182, ISSN 0377-0427, doi: http://dx.doi.org/10.1016/j.cam.2009.08.077.

[20] A. L. Kalamkarov, I. V. Andrianov, V. V. Danishevskyy, Asymptotic Homogenization of Composite Materials and Structures, Applied Mechanics Reviews 62 (3) (2009) 030802, ISSN 00036900, doi:10.1115/1.3090830.

[21] R. Hill, Elastic properties of reinforced solids: Some theoretical principles, Journal of the Mechanics and Physics of Solids 11 (5) (1963) $357-372$, ISSN 0022-5096, doi:http://dx.doi.org/10.1016/0022-5096(63)90036-X.

[22] M. Ostoja-Starzewski, X. Wang, Stochastic finite elements as a bridge between random material microstructure and global response, Computer Methods in Applied Mechanics and Engineering 168 (14) (1999) 35 - 49, ISSN 0045-7825, doi:http://dx.doi.org/10.1016/S0045-7825(98)00105-4. 
[23] J. Fish, W. Wu, A nonintrusive stochastic multiscale solver, International Journal for Numerical Methods in Engineering 88 (9) (2011) 862-879, ISSN 1097-0207, doi:10.1002/nme.3201.

[24] V. Gusella, F. Cluni, Random field and homogenization for masonry with nonperiodic microstructure, Journal of Mechanics of Materials and Structures 1 (2) (2006) 357-386, ISSN 1559-3959, doi:10.2140/jomms.2006.1.357.

[25] P. Trovalusci, M. Ostoja-Starzewski, M. L. De Bellis, A. Murrali, Scaledependent homogenization of random composites as micropolar continua, European Journal of Mechanics - A/Solids 49 (0) (2015) 396 - 407, ISSN 0997-7538, doi:http://dx.doi.org/10.1016/j.euromechsol.2014.08.010.

[26] X. Yin, W. Chen, A. To, C. McVeigh, W. Liu, Statistical volume element method for predicting microstructureconstitutive property relations, Computer Methods in Applied Mechanics and Engineering 197 (43 - 44) (2008) 3516 - 3529, ISSN 0045-7825, doi: http://dx.doi.org/10.1016/j.cma.2008.01.008, stochastic Modeling of Multiscale and Multiphysics Problems.

[27] X. Yin, S. Lee, W. Chen, W. K. Liu, M. F. Horstemeyer, Efficient Random Field Uncertainty Propagation in Design Using Multiscale Analysis, Journal of Mechanical Design 131 (2), ISSN 1050-0472, doi: http://dx.doi.org/10.1115/1.3042159.

[28] A. Clément, C. Soize, J. Yvonnet, Computational nonlinear stochastic homogenization using a nonconcurrent multiscale approach for hyperelastic heterogeneous microstructures analysis, International Journal for Numerical Methods in Engineering 91 (8) (2012) 799-824, ISSN 1097-0207, doi: 10.1002/nme.4293.

[29] C. Soize, Maximum entropy approach for modeling random uncertainties in transient elastodynamics, The Journal of the Acoustical Society of America 109 (5) (2001) 1979-1996, doi:http://dx.doi.org/10.1121/1.1360716.

[30] C. Soize, Random matrix theory for modeling uncertainties in computational mechanics, Computer Methods in Applied Mechanics and Engineering 194 (12 - 16) (2005) 1333 - 1366, ISSN 0045-7825, doi: http://dx.doi.org/10.1016/j.cma.2004.06.038, special Issue on Computational Methods in Stochastic Mechanics and Reliability Analysis.

[31] S. Das, R. Ghanem, A Bounded Random Matrix Approach for Stochastic Upscaling, Multiscale Modeling \& Simulation 8 (1) (2009) 296-325, doi: 10.1137/090747713.

[32] J. Guilleminot, A. Noshadravan, C. Soize, R. Ghanem, A probabilistic model for bounded elasticity tensor random fields with application to polycrystalline microstructures, Computer Methods in Applied Mechanics and Engineering 200 (17 - 20) (2011) 1637 - 1648, ISSN 0045-7825, doi:http://dx.doi.org/10.1016/j.cma.2011.01.016. 
[33] A. Noshadravan, R. Ghanem, J. Guilleminot, I. Atodaria, P. Peralta, Validation of a probabilistic model for mesoscale elasticity tensor or random polycrystals, International Journal for Uncertainty Quantification 3 (1) (2013) 73-100, ISSN 2152-5080.

[34] H. Cho, D. Venturi, G. Karniadakis, KarhunenLove expansion for multi-correlated stochastic processes, Probabilistic Engineering Mechanics 34 (0) (2013) 157 - 167, ISSN 0266-8920, doi: http://dx.doi.org/10.1016/j.probengmech.2013.09.004.

[35] M. Shinozuka, Simulation of Multivariate and Multidimensional Random Processes, The Journal of the Acoustical Society of America 49 (1B) (1971) 357-368, doi:http://dx.doi.org/10.1121/1.1912338.

[36] M. Shinozuka, C.-M. Jan, Digital simulation of random processes and its applications, Journal of Sound and Vibration 25 (1) (1972) 111 - 128, ISSN 0022-460X, doi:http://dx.doi.org/10.1016/0022-460X(72)90600-1.

[37] C. Soize, Identification of high-dimension polynomial chaos expansions with random coefficients for non-Gaussian tensor-valued random fields using partial and limited experimental data, Computer Methods in Applied Mechanics and Engineering 199 (33 - 36) (2010) 2150 - 2164, ISSN 0045-7825, doi:10.1016/j.cma.2010.03.013.

[38] A. Der Kiureghian, J. Ke, The stochastic finite element method in structural reliability, Probabilistic Engineering Mechanics 3 (2) (1988) 83 - 91, ISSN 0266-8920, doi:http://dx.doi.org/10.1016/0266-8920(88)90019-7.

[39] S. Lepage, Stochastic finite element method for the modeling of thermoelastic damping in micro-resonators, Leloup, 2007.

[40] C. Huet, Application of variational concepts to size effects in elastic heterogeneous bodies, Journal of the Mechanics and Physics of Solids 38 (6) (1990) 813 - 841, ISSN 0022-5096, doi:http://dx.doi.org/10.1016/00225096(90)90041-2.

[41] V.-D. Nguyen, E. Béchet, C. Geuzaine, L. Noels, Imposing periodic boundary condition on arbitrary meshes by polynomial interpolation, Computational Materials Science 55 (2012) 390-406, doi: 10.1016/j.commatsci.2011.10.017.

[42] S. Hazanov, C. Huet, Order relationships for boundary conditions effect in heterogeneous bodies smaller than the representative volume, Journal of the Mechanics and Physics of Solids 42 (12) (1994) 1995 - 2011, ISSN 0022-5096, doi:http://dx.doi.org/10.1016/0022-5096(94)90022-1.

[43] M. Hopcroft, W. Nix, T. Kenny, What is the Young's Modulus of Silicon?, Microelectromechanical Systems, Journal of 19 (2) (2010) 229-238, ISSN 1057-7157, doi:10.1109/JMEMS.2009.2039697. 
[44] M. G. D. Geers, E. W. C. Coenen, V. G. Kouznetsova, Multi-scale computational homogenization of structured thin sheets, Modelling and Simulation in Materials Science and Engineering 15 (4) (2007) S393, doi:10.1088/09650393/15/4/S06.

[45] M. P. Sena, M. Ostoja-Starzewski, L. Costa, Stiffness tensor random fields through upscaling of planar random materials, Probabilistic Engineering Mechanics 34 (2013) 131-156, doi:10.1016/j.probengmech.2013.08.008.

[46] J. Reddy, On locking-free shear deformable beam finite elements, Computer Methods in Applied Mechanics and Engineering 149 (1 - 4) (1997) 113 132, ISSN 0045-7825, doi:http://dx.doi.org/10.1016/S0045-7825(97)000753 , containing papers presented at the Symposium on Advances in Computational Mechanics.

[47] S. Mulay, G. Becker, R. Vayrette, J.-P. Raskin, T. Pardoen, M. Galceran, S. Godet, L. Noels, Multiscale modelling framework for the fracture of thin brittle polycrystalline films: application to polysilicon, Computational Mechanics 55 (2015) 73-91, ISSN 0178-7675, doi:10.1007/s00466-014-10834 .

[48] C. Geuzaine, J.-F. Remacle, Gmsh: A 3-D finite element mesh generator with built-in pre- and post-processing facilities, International Journal for Numerical Methods in Engineering 79 (11) (2009) 1309-1331, doi: 10.1002/nme.2579.

[49] C. Soize, Non-Gaussian positive-definite matrix-valued random fields for elliptic stochastic partial differential operators, Computer Methods in Applied Mechanics and Engineering 195 (13) (2006) 26 - 64, ISSN 0045-7825, doi:http://dx.doi.org/10.1016/j.cma.2004.12.014.

[50] R. Popescu, G. Deodatis, J. Prevost, Simulation of homogeneous nonGaussian stochastic vector fields, Probabilistic Engineering Mechanics 13 (1) (1998) 1 - 13, ISSN 0266-8920, doi:10.1016/S0266-8920(97)00001-5. 